\title{
Comparative study of immunohematological tests with canine blood samples submitted for a direct antiglobulin (Coombs') test
}

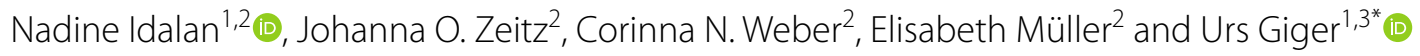

\begin{abstract}
Background: A 2019 ACVIM consensus statement on diagnostics for immune-mediated hemolytic anemia (IMHA) in dogs made testing recommendations. As data on the performance of immunohematological tests was lacking, we undertook a comparative analysis.

Material and methods: Anticoagulated blood samples from 126 dogs suspected of having IMHA submitted to a diagnostic veterinary laboratory for a routine direct antiglobulin test (DAT) and from 28 healthy control dogs were evaluated for spherocytosis and autoagglutination before and after three saline washes. Samples were also subjected to different DATs: a gel minitube and an immunochromatographic strip kit used in clinics; neutral gel column cards, microtiter plates (at $4^{\circ}, 22^{\circ}$, and $37^{\circ} \mathrm{C}$ ), capillary tubes, and flow cytometry used in laboratories.

Results: Samples from healthy dogs yielded negative results with all immunodiagnostic tests. Among the 126 samples submitted for DAT 67 were positive by a DAT utilizing microtiter plates with goat anti-dog antiglobulin DAT at $22^{\circ} \mathrm{C}$. Notably, DAT results were comparable and consistent across all evaluated methods regardless of antiglobulin and temperature used. DAT+ dogs were more severely anemic and more likely to have erythroid regeneration compared to DAT- dogs. Macroscopic agglutination in tubes or on slides was observed in 48 samples after 1:1 and 1:4 blood to saline dilution, but only persisted in four samples after washing. Among the DAT+ samples, $57 \%$ had agglutination, $87 \%$ had spherocytosis, and $45 \%$ had both. There was good correlation between spherocytosis and DAT results from the six DAT techniques, but the correlation with autoagglutination was only fair. Clinical follow-up was available for 42 dogs. Of the sample from 12 DAT+ dogs collected during treatment, 10 remained DAT+ when tested 1-24 weeks after initial assessment.
\end{abstract}

Conclusions: Based upon this comparative prospective survey, all in-clinic and laboratory DAT techniques produced similar results when performed by trained personnel and can therefore be recommended for detection of antibodycoated erythrocytes and immunohematological diagnosis. In addition, use of these tests for monitoring response of IMHA dogs to treatment might be valuable.

Keywords: Autoagglutination, Spherocytes, Erythrocytes, Dog, Canine, Immune-mediated hemolytic anemia

*Correspondence: giger@upenn.edu

${ }^{1}$ Vetsuisse Faculty, University of Zürich, Winterthurerstrasse 260,

8057 Zürich, Switzerland

Full list of author information is available at the end of the article

(c) The Author(s). 2021, corrected publication 2021. Open Access This article is licensed under a Creative Commons Attribution 4.0 International License, which permits use, sharing, adaptation, distribution and reproduction in any medium or format, as long as you give appropriate credit to the original author(s) and the source, provide a link to the Creative Commons licence, and indicate if changes were made. The images or other third party material in this article are included in the article's Creative Commons licence, unless indicated otherwise in a credit line to the material. If material is not included in the article's Creative Commons licence and your intended use is not permitted by statutory regulation or exceeds the permitted use, you will need to obtain permission directly from the copyright holder. To view a copy of this licence, visit http://creativecommons.org/licenses/by/4.0/. The Creative Commons Public Domain Dedication waiver (http://creativecommons.org/publicdomain/zero/1.0/) applies to the data made available in this article, unless otherwise stated in a credit line to the data. 


\section{Plain English summary}

Immune-mediated hemolytic anemia (IMHA) is driven by accelerated destruction of antibody-coated red blood cells (RBCs). The clinical diagnosis of IMHA requires blood tests and can be challenging due to the lack of established reference methods and reagents. We compared routine and specific blood test methods in samples from 126 dogs suspected of having IMHA. In addition, 28 samples from healthy non-anemic dogs served as negative controls. Using a variety of direct antiglobulin (Coombs') test (DAT) methods, antibody-coated RBCs were detected in approximately half of the samples from dogs with suspected IMHA. The results of four laboratory and two in-clinic DAT kits were comparable and consistent. Marked spherocytosis (small spherical RBCs) and to a lesser extend autoagglutination (clumping of RBCs) also correlated with the DAT results. Furthermore, dogs with DAT positive results were more severely anemic and more likely to have active bone marrow responses compared to dogs with negative DAT results. These concordant results underscore the diagnostic value of both laboratory and in-clinic DAT methods for IMHA in dogs. They confirm marked spherocytosis and a positive DAT result as useful clinical parameters for diagnosing and potentially monitoring this disease.

\section{Background}

Immune-mediated hemolytic anemia (IMHA) is driven by accelerated destruction of antibody-coated red blood cells (RBCs). Approaches to the diagnosis of IMHA in dogs remain controversial, with some investigators and practicing clinicians applying strict hematological and immunological parameters, while others use a combination of RBC agglutination, spherocytosis or responsiveness to immunosuppressive therapy as diagnostic criteria. For many years, we have recommended that IMHA be diagnosed by evidence of in vivo hemolysis and one of three specific immunohematological test results: persistent autoagglutination after three saline washes, marked spherocytosis, and/or a positive DAT result [1-5].

In 2019, the American College of Veterinary Internal Medicine (ACVIM) consensus statement offered similar guidelines, but the data substantiating these recommendations were sparse [6]. There remain disparities regarding the routinely used diagnostic techniques and interpretations of specific test results for diagnosis of IMHA in veterinary clinics as well as in laboratories, as recently illustrated in a small survey by the Veterinary and Comparative Clinical Immunology Society [7].

In human medicine, a positive Coombs' test result is required for the diagnosis of IMHA [8, 9]. The Coombs' test was established in 1945 by Robin Coombs, a veterinarian and immunologist at Cambridge, United
Kingdom, although Italian scientists reported on the conceptual basis for this test even earlier [10]. The DAT detects bound antibodies (and complement) on the surface of erythrocytes [11, 12]. Species-specific antiglobulins (also known as Coombs' reagent) are used to detect bound antibodies and/or complement (C3) by an agglutination or other erythrocytic binding reaction. Originally performed as a tube assay, a variety of DAT methods are currently utilized in human diagnostic laboratories, including microtiter plate, capillary, gel minitube, and flow cytometric technologies $[8,13-15]$. However, there are no single reference (gold standard) DAT methods or specific antiglobulin reagents established $[8,9,16]$, and in-clinic DAT kits are not available in human medicine. Notably, the nature of the antigens to which the autoantibodies bind on the erythrocyte membrane is still not precisely defined $[8,9,14,17]$.

In contrast to human medicine, the DAT is not universally available in canine medicine, and the value of DAT results has been questioned by veterinary clinicians, due to concerns with false negative and false positive DAT results, that are potentially related to ineffective reagents, faulty methods, incorrect test interpretation, and interference by treatments, such as immunosuppressive drugs and transfusions $[1-3,6,8,18]$. It is the authors' impression that the DAT is often skipped in the diagnostic approach of IMHA in dogs. Indeed, in a recent study of dogs suspected of having IMHA, only $20 \%$ were tested by DAT [19]. As such, a presumptive diagnosis of IMHA is frequently made without evidence of immune-mediated destruction of erythrocytes in anemic dogs $[1-3,7,20$, 21].

The senior author's (UG) laboratory had introduced and compared several in-clinic and laboratory DAT methods to diagnose IMHA in a small number of dogs in 2014 [3]. In the present study, prior investigations were expanded to include samples from a larger cohort of dogs submitted to a diagnostic laboratory for DAT, and also from healthy control dogs by comparing various, in part novel, means to document erythrocytic immune destruction. In addition to evaluation of marked spherocytosis, saline agglutination test (SAT), and persistent autoagglutination after washing, the presence of antibody-coated erythrocytes was documented by two in-clinic and four laboratory DAT techniques.

\section{Materials and methods \\ Blood samples from dogs}

Ethylenediaminetetraacetic acid (EDTA)-anticoagulated blood samples from dogs suspected of having IMHA and for which a DAT was requested were gathered at a major veterinary diagnostic laboratory (Laboklin, GmbH\&Co.KG, Bad Kissingen, Germany). Samples with 
DAT positive (DAT+) or DAT negative (DAT-) results by routine laboratory methods were included, if the leftover sample was $\geq 500 \mu \mathrm{L}$ and the sample was kept at $4{ }^{\circ} \mathrm{C}$ for $<6$ days prior to investigation. During the study, the routinely performed DAT methods used at the diagnostic laboratory were changed, and, thus, those results were not included. Samples were only included when the veterinarian who performed all tests on each sample (NI), had time to perform these laboratory analyses (requiring about four hours $[\mathrm{h}]$ ). All veterinarians who submitted samples that were DAT+ results were contacted to obtain information about the dog's clinical signs in support of a IMHA diagnosis and any available clinical follow-up. They were also offered free follow-up laboratory testing. Despite reminders, clinical information could not be obtained from all dogs. However, for some DAT+ cases, follow-up EDTA blood samples were received one week to six months after the first DAT+ results (initial presentation) to assess response to treatment and resolution of the immune destruction of erythrocytes.

In addition, blood samples from dogs with normal routine blood test results (a complete blood count [CBC] and serum chemistry panel) performed as part of a health screen were selected from samples submitted to the same laboratory (without a request for a routine DAT) and served as negative controls. Some of these samples were also blood typed [22] and used to induce in vitro DAT + controls by adding anti-dog erythrocyte antigen (DEA) 4, anti-DEA 5, or anti-Dal alloantibodies to coat erythrocytes (see below).

Signalment and routine blood test results $(\mathrm{CBC}$ and serum chemistry panel), performed at Laboklin or submitted along with blood samples from the clinics, were gathered from each dog. Only serum samples separated from the cellular components before shipment were included in the bilirubin assessment. The use of left-over blood from the samples routinely submitted for diagnostics at Laboklin was approved by the governmental animal care and committee in Bayern, Germany.

\section{Laboratory techniques}

For each sample, microscopic blood smear examinations, various agglutination tests, and DATs were performed with either whole blood or after centrifugation and washing within $4 \mathrm{~h}$ as outlined (Suppl Table S1) according to either manufacturer's instructions or standard operating procedures. The overnight incubation of the microtiter plate at $4^{\circ} \mathrm{C}$ required an additional time period (12-15 h).

\section{Sample preparation}

Initially, each blood sample was visually examined for gross autoagglutination in the submitted EDTA tube and graded semi-quantitatively as 0 (no clumps), 1+(fine clumps $[\leq 1 \mathrm{~mm}])$, or $2+($ granular clumps $[>1 \mathrm{~mm}])$. Tubes were then centrifuged at $240 \times \mathrm{g}$ for 5 minutes (min), and after visual inspection, the plasma was separated from packed red blood cells (pRBCs). Plasma appearance was categorized as normal, lipemic, icteric, or weakly to strongly hemolytic from 0 to $3+: 0$ for transparent straw-colored, $1+$ for a slight pink coloration, $2+$ for a bright red coloration, and $3+$ for a dark red discoloration [23].

\section{Hemoglobin and packed cell volume}

The required amount of EDTA blood and red blood cells (RBCs) for all DATs, agglutination tests, and blood smears was estimated in $\mu \mathrm{L}$ based on the blood's hemoglobin $(\mathrm{Hb})$ content $\left(\mathrm{HemoCue}^{\circledR} \mathrm{Hb} 210+[\right.$ Brea, CA, USA] or from $\mathrm{CBC}$ ). When additional EDTA blood was available beyond what was needed for the various tests related to immune destruction of RBCs (see below), the microhematocrit (packed cell volume $[\mathrm{PCV}]$ ), and plasma $\mathrm{Hb}\left(\mathrm{HemoCue}^{\circledR}\right)$ concentration were measured. When both total blood $\mathrm{Hb}$ and PCV values were available, the blood $\mathrm{Hb}$ concentration (in $\mathrm{g} / \mathrm{dL}$ ) was multiplied by three to express the predicted hematocrit (Hct) in percent for comparison.

\section{Autoagglutination testing before and after washing}

Independent of the degree of visual tube autoagglutination, either one or four drops of $0.9 \% \mathrm{NaCl}$ solution were mixed with one drop of blood to assess autoagglutination macroscopically on separate microscopic slides (1:1 and 1:4 SAT) [3]. Both the microscopic slides and saline were kept at $22^{\circ} \mathrm{C}$ (room temperature), and blood samples were brought to $22^{\circ} \mathrm{C}$ prior to testing.

All samples which showed any autoagglutination in the tube and/or on the slide were further tested for persistence of (also referred to as true) autoagglutination after three saline washes [3]. Briefly, one part pRBCs obtained by centrifugation was resuspended in approximately four parts of saline and then centrifuged at $240 \times \mathrm{g}$ for $5 \mathrm{~min}$. The supernatant was discarded and the process was repeated twice more. Then one drop of washed pRBCs was mixed on a slide with four drops of saline. The autoagglutination was assessed macroscopically and categorized as present or absent.

\section{Microscopic examination of blood smears for spherocytosis, autoagglutination, and ghost cells}

Blood smears were prepared 1-5 days after blood collection and were performed along with other tests. Blood smears were fixed and stained (Wright Giemsa stain modified, Sigma-Aldrich, Taufkirchen, Germany) and microscopically examined. Spherocytosis was considered present when $\geq 5$ spherocytes were seen in microscopic 
fields with a $100 \times$ high-power field (hpf) $[3,24,25]$. The presence of $\geq 5$ ghost cells $/ 100 \times \mathrm{hpf}$ was also noted [17, $26]$. Finally, the presence of polychromasia $(1+: 2-7,2+$ : 8-14, 3+: 15-29, 4+: > 30 cells/1000 $\times$ microscopic monolayer field) and rouleaux formation was assessed on blood smears as present or absent.

\section{Direct antiglobulin tests}

Erythrocyte-bound antibodies were detected using six DAT techniques with different polyclonal antiglobulin reagents, incubation periods, and temperatures (Suppl Table S1 and Suppl Figure S1). Prior to this prospective laboratory study, protocols for the various DAT techniques were established based upon manufacturers' instructions and literature, but no formal validation studies were done for any assays. Polyclonal anti-DEA 4 and anti-DEA 5 (ABRI, Animal Blood Resources International, Dixon, California) as well as anti-Dal (from clinically sensitized dog [22]) alloantibodies were used to induce in vitro positive controls for neutral gel column card (GEL LAB), microcapillary tube (CAPIL), and microtiter plate (MICRO) DAT. Anti-Dal was used undiluted, while anti-DEA 4 and anti-DEA 5 were diluted 1:8 and $1: 2.5$, respectively [22].

Immunochromatographic strip kit (STRIP KIT) DAT method The immunochromatographic strip kit DAT (STRIP KIT DAT; Canine Labtest DAT®, Alvedia, Limonest, France) was performed according to the manufacturer's instructions and as previously described [3]. Since the original description of the STRIP KIT DAT [3], the erythrocytic binding strength at the band, where the antiglobulin (RAD: rabbit anti-dog IgG, IgM, and C3 canine anti-globulin Coombs' reagent, MP Biomedicals, Solon, OH, USA) is located, has been enhanced according to the manufacturer. The test results were graded by visual inspection as negative (-) and weakly to strongly positive $(1+$ to $4+)$ based on the intensity of the observed antiglobulin test band compared to the strength of the control band. If RBCs stayed at the origin of the strip, it was considered to be due to autoagglutination and thus recorded as a strongly positive result as per manufacturer's instructions.

Gel minitube kit (GEL KIT) DAT method The gel minitube kit DAT (GEL KIT DAT, Gel Test Canine DAT $\odot$, Alvedia) was performed following the manufacturer's instructions and using a recommended centrifuge with swinging buckets (Hettich EBA 270, Tüttlingen, Germany). While RBC washing is not required, this test was performed twice with a whole blood suspension ( $5 \mu \mathrm{L}$ whole blood in $495 \mu \mathrm{L}$ buffer solution) and with washed RBCs in separate minitubes containing the antiglobulin (RAD) in gel. The test results were graded as negative $(-), 1+, 2+, 3+$, and $4+$, as described by the manufacturer and previous gel column and tube studies $[3,13,15,20]$. The neutral gel minitubes (Neutral gel test, Alvedia) were used as additional auto-controls beside the neutral gel column test (see below; both gels lacked incorporated antiglobulins), and results were negative.

Laboratory gel column card (GEL LAB) DAT method with neutral gel columns Each blood sample was tested by the gel column card DAT method with neutral gel columns (GEL LAB DAT) as previously described [3] after $\mathrm{RBC}$ washing with saline (negative auto-control) and with one of two different polyclonal canine antiglobulin reagents: GAD (goat anti-dog IgG, IgM, and C3: Canine Coombs Reagent, VMRD, Pullman, WA, USA) or RAD (MP Biomedicals). Briefly, a $1 \%$ RBC suspension was prepared by mixing $5 \mu \mathrm{L}$ pRBCs in $500 \mu \mathrm{L}$ saline. Then, 13 $\mu \mathrm{L}$ RBC suspension was mixed with $6.3 \mu \mathrm{L}$ GAD, RAD, or saline (i.e., a 1:2 ratio of antiglobulin solution to $R B C$ suspension) on top of neutral gel columns ( $\mathrm{NaCl}$ Enzyme Test and Cold Agglutinin ID-card, DiaMed $\mathrm{GmbH}$, Cressier, Switzerland). The gel column cards were incubated for $15 \mathrm{~min}$ at $37^{\circ} \mathrm{C}$ (Incubator IN30, Memmert, Germany) and, thereafter, centrifuged in a special centrifuge (Diamed ID-Centrifuge 6S, DiaMed) for $10 \mathrm{~min}$ at $85 \times \mathrm{g}$. The test results were interpreted as for the GEL KIT DAT described above [3, 13]. Care must be taken to avoid the presence of air between the reagent and the red blood cell suspension, as incorrectly mixed solutions might falsify the test result. The problem can be easily solved by gently shaking the sample column until the suspension is mixed and the locked air escapes.

Capillary (CAPIL) DAT method The capillary (CAPIL) DAT was performed as described [3, 13] with both (GAD and RAD) antiglobulins. Briefly, one third of a microcapillary tube (Precision capillary tube $0.4 \times 75 \mathrm{~mm}$, Drummond Scientific Company, Broomall, PA, USA) was filled with one of the two antiglobulin reagents, and a $30 \%$ washed RBC suspension in saline was added until two thirds of the microcapillary tube was reached. The microcapillary tube was then inverted, incubated in a $60^{\circ}$ angle in clay at $22^{\circ} \mathrm{C}$ for $10 \mathrm{~min}$, and the visual presence $(+)$ or absence (-) of agglutination was then noted. As also observed with the LAB GEL DAT method, air trapped between the blood and the reagent can become an issue by keeping the reagent and the blood apart. This technical issue can be easily solved by gently tapping the microcapillary tube to allow the trapped air to escape $[3,27]$.

Laboratory microtiter plate (MICRO) DAT method For the microtiter plate (MICRO) DAT method, a $4 \%$ RBC 
suspension was produced by adding $14.4 \mu \mathrm{L}$ washed pRBCs to $346 \mu \mathrm{L}$ saline. An 11-step doubling dilution gradient from 1:2 to 1:2048 with each of the two canine antiglobulin reagents described above (GAD [MICRO GAD DAT] and RAD [MICRO RAD DAT]) was set up in a 96-well round bottom microtiter plate (Microtiter plate 96 wells U-Form, Merck, Darmstadt, Germany), as described [3, 28, 29]. To the last well of each horizontal row, $15 \mu \mathrm{L}$ saline was added as a negative (or autoagglutination) control. The microtiter plate was incubated (Incubator IN30) at three different temperatures: $22^{\circ} \mathrm{C}$ for $30 \mathrm{~min}$, then $37^{\circ} \mathrm{C}$ for $30 \mathrm{~min}$, and, finally, at $4^{\circ} \mathrm{C}$ overnight for 12-15 h. Between each temperature change, the agglutination test result was recorded, and any agglutination was completely dispersed by horizontally shaking of the plate before each re-incubation. Each row was evaluated for the presence $(+)$ or absence (-) of agglutination, and the degree of positivity was based upon the last dilution with observed agglutination from 1:2 to 1:2048 [3]. While all DAT methods were compared to each other, the result presentation focuses on the comparison to the MICRO GAD DAT at $22^{\circ} \mathrm{C}$.

Laboratory flow cytometric (FLOW) DAT method The flow cytometric (FLOW) DAT was performed with fluorescein isothiocyanate (FITC)-marked goat anti-dog IgG antibodies (Goat anti-dog IgG $[\mathrm{H}+\mathrm{L}]$ :FITC, BioRad, Oxfordshire, United Kingdom) and an Attune NxT flow cytometer (Thermo Fisher, Waltham MA, USA) as described [18]. Briefly, $5 \mu \mathrm{L}$ pRBCs were washed three times with $500 \mu \mathrm{L}$ phosphate-buffered saline (PBS) containing $0.5 \%$ bovine serum albumin $(0.5 \%$ BSA, FACS Cell Wash, Becton Dickinson GmbH, Heidelberg, Germany, named BSA-PBS) at $200 \mathrm{x}$ g for $15 \mathrm{~min}$. Then, 100 $\mu \mathrm{L}$ of a $1 \%$ washed $\mathrm{RBC}$ suspension were mixed with 100 $\mu \mathrm{L}$ of the 1:10 diluted antiglobulin reagent and incubated in the dark at $4^{\circ} \mathrm{C}$ for $30 \mathrm{~min}$. The suspension was washed again three times after incubation and finally resuspended in $200 \mu \mathrm{L} \mathrm{PBS} / 0.5 \%$ BSA before measurement. The scatter plot's side scatter height $(\mathrm{SSC}-\mathrm{H})$ versus forward scatter height (FSC-H), forward scatter width versus (FSC-W) versus FSC-H were applied for gating, and $\mathrm{SSC}-\mathrm{H}$ versus FITC-H were used to assess the degree of positivity for each sample. A washed $1 \%$ RBC suspension without antibodies was used as negative control. For each sample, 10,000 events were recorded in a pre-gated region. The sample positivity was stated as percentage of all gated RBCs. All measurements were performed with a FSC wavelength of $240 \mathrm{~nm}$ and SSC of $380 \mathrm{~nm}$. Blood samples of 12 clinically healthy dogs were used to establish reference values. Values of $>2$ standard deviations (SD) above the mean percentage of positive cells were considered DAT + .

\section{Data analyses}

The entire dataset of samples submitted for a routine DAT were divided into DAT+ and DAT- groups. Samples were considered DAT + when they were scored positive by the laboratory MICRO GAD DAT results or scored positive using 4-6 DAT methods (named $\geq 4$ DAT + ). Samples were considered DAT- when either the MICRO GAD DAT result was negative or fewer than three other DAT method results were positive.

All statistical analyses were performed with SPSS Statistics 25 software [30]. Cohen's and Fleiss' kappa [31] ( $\mathrm{k}$ )-values and two-sided 95\% confidence intervals $(95 \%$ $\mathrm{CI})$ were calculated to assess the degree of agreement with all possible pairs of DAT methods. $\mathrm{K}$-value analyses were performed twice: first using samples from dogs submitted for DAT, and thus suspected of having IMHA by the attending veterinarian, and also by including the 28 healthy control dogs, and interpreted with the scale originally established by Landis and Koch [32], with modified category names [33]. A $p$-value of $<0.05$ was considered significantly different. The normality assumption was tested with a Shapiro-Wilk test, histograms, box plots and Q-Q diagrams [34]. If not met, a Mann-Whitney $\mathrm{U}$ test was performed instead of a t-test $[35,36]$. The nominal data were analyzed with chi-squared test or, if not indicated due to small number of samples, a Fisher's exact test [37, 38]. In addition, the analytical diagnostic sensitivity and specificity were calculated for each method in comparison to the MICRO DAT with GAD at $22^{\circ} \mathrm{C}[39,40]$.

\section{Results}

From November 2019 until November 2020, EDTAanticoagulated blood samples from 126 dogs suspected of having IMHA and for which a routine DAT was requested were analyzed at a major veterinary diagnostic laboratory (Laboklin GmbH\&Co.KG, Bad Kissingen, Germany). The blood samples came mainly from Germany but also from a few other European countries. Because of sample limitations (insufficient quantity; too old) and/or operator availability (first author [NI] was not always available to perform extensive testing), an additional $78 \mathrm{DAT}+$ and 1,033 DAT- samples submitted for routine DAT during the same 13-month period were not included.

\section{Healthy negative control dogs and positive control samples}

Additional EDTA blood samples submitted from a total of 28 healthy dogs were included in this survey. None of them showed anemia, autoagglutination, spherocytosis, or positive DAT results by any of the test methods performed. This DAT- control group was composed of 20 
mixed breed and eight purebred dogs with a female to male ratio of 1.55 , a median age of nine years, and age range of 1-14 years (Suppl Table S2 and S3, A to C).

In addition, samples from eight of the above 28 negative control dogs were examined after their blood was incubated with polyclonal anti-DEA 4, anti-DEA 5, or anti-Dal to allow coating of RBCs, thereby serving as DAT+ control groups. All of these dogs had the common blood type pattern $D E A 4+, D E A$ 5-, and Dal+ [22]. Exposure to anti-DEA 4 and anti-Dal induced a positive DAT reaction in all eight dogs with all DAT methods applied, but the degree of positivity differed depending on the dog tested. As expected by their blood type, anti-DEA 5 did not produce DAT + results in these dogs (Suppl Table S4). Together, these results provide appropriate negative and positive controls which were mostly lacking in previous studies.

\section{Signalment, blood $\mathrm{Hb}$ concentration, and $\mathrm{CBC}$}

In the 126 dogs for which a routine DAT was clinically requested, 44 were mixed breed and 78 were purebred dogs with no more than six dogs per breed (breed was not stated for four dogs) and more females than males (ratio 1.41). Neutering status was infrequently mentioned and thus is not reported here. The age ranged between 0.5 to 15 years, with a median of eight years. When comparing the signalment of DAT+against DAT- dogs, no significant differences were observed, except there were more females in the MICRO GAD DAT+than DATgroup (Suppl Table S2 and S3 A to C).

Based upon measurements of total blood $\mathrm{Hb}$ concentrations and $\mathrm{CBC}$ results, 105 of 126 tested dogs were mildly to severely anemic. The DAT+dogs were more frequently and more severely anemic and more likely to have hyperbilirubinemia than the DAT- dogs (Suppl Table S5 and S6, Suppl Figure S2A, D and S3A,
D). More than half of tested dogs showed a reticulocytosis $(>110,000 / \mathrm{nL})$ and $65 \%$ of them were DAT+ with the MICRO GAD DAT method and $\geq 4$ DAT+, respectively. The degree of reticulocytosis correlated with microscopically observed polychromasia, but not with the degree of anemia (Suppl Table S5 and S7, Suppl Figure $\mathrm{S} 2 \mathrm{C}$ and $\mathrm{S} 3 \mathrm{C}$ ). The presence of $\mathrm{Hb}$ in plasma samples did not differ significantly in DAT+ and DAT- dogs (Suppl Table S5, Suppl Figure S2B and S3B).

\section{Assessment of blood smear}

Ghost cells, as proposed by ACVIM consensus report, were only rarely found and showed a slight association with the $\geq 4$ DAT+ group, but not with the MICRO GAD DAT+results (Table 1). Marked spherocytosis was seen frequently and significantly more often in DAT+ than DAT- dogs (Table 1). Only one of 58 dogs with spherocytosis was DAT- by all methods. The presence of rouleaux formation did not differ significantly between the DAT+ and DAT- groups and was not associated with the presence of agglutination (Table 1).

\section{Evaluation for macroscopic autoagglutination}

Autoagglutination of EDTA blood in tubes was visually observed more often in samples from DAT+dogs than from DAT- dogs (Table 1). All samples with visual tube agglutination also showed macroscopic agglutination by SAT in undiluted blood or after 1:1 or 1:4 saline dilution. Ten additional samples showed macroscopic autoagglutination in SAT without tube agglutination (Table 1). Agglutination was noted in 48 samples with $\sim 30 \%$ having a granular pattern, and the remainder exhibiting only fine agglutination. Finally, after washing blood three times with saline, only four

Table 1 Spherocytosis, ghost cells, rouleaux, and macroscopic agglutination test results compared to DAT results in 126 dogs suspected to have IMHA

\begin{tabular}{|c|c|c|c|c|c|c|c|c|c|c|c|c|c|}
\hline \multirow[t]{2}{*}{ Test group } & \multirow[t]{2}{*}{ DAT result } & \multicolumn{2}{|c|}{ Spherocytosis ${ }^{a}$} & \multicolumn{2}{|c|}{ Ghost cells $^{\mathrm{a}}$} & \multicolumn{2}{|c|}{ Rouleaux $^{a}$} & \multicolumn{2}{|c|}{ SAT 1:1 } & \multicolumn{2}{|c|}{ SAT 1:4 } & \multicolumn{2}{|c|}{$\begin{array}{c}\text { Persistent } \\
\text { agglutination }\end{array}$} \\
\hline & & Yes & No & Yes & No & Yes & No & Yes & No & Yes & No & Yes & No \\
\hline \multirow[t]{3}{*}{ MICRO DAT+ } & Yes & 54 & 8 & 10 & 52 & 13 & 49 & 38 & 29 & 38 & 29 & 4 & 63 \\
\hline & No & 4 & 54 & 3 & 55 & 13 & 45 & 10 & 49 & 10 & 49 & 0 & 59 \\
\hline & pvalue & \multicolumn{2}{|c|}{$<.001$} & \multicolumn{2}{|c|}{.077} & \multicolumn{2}{|c|}{1.0} & \multicolumn{2}{|c|}{$<.001$} & \multicolumn{2}{|c|}{$<.001$} & \multicolumn{2}{|c|}{.122} \\
\hline \multirow[t]{3}{*}{$\geq 4$ DAT+ } & Yes & 53 & 9 & 11 & 51 & 14 & 48 & 39 & 28 & 39 & 28 & 4 & 63 \\
\hline & No & 5 & 53 & 2 & 56 & 12 & 46 & 9 & 50 & 9 & 50 & 0 & 59 \\
\hline & pvalue & \multicolumn{2}{|c|}{$<.001$} & \multicolumn{2}{|c|}{.017} & \multicolumn{2}{|c|}{0.828} & \multicolumn{2}{|c|}{$<.001$} & \multicolumn{2}{|c|}{$<.001$} & \multicolumn{2}{|c|}{.122} \\
\hline
\end{tabular}

Groups were compared by chi-squared test

$S A T$ saline agglutination test, $D A T$ direct antiglobulin test, MICRO DAT microtiter plate DAT with goat anti-dog $\operatorname{lgG}, \lg \mathrm{M}$, and $C 3$ reagent incubated at $22^{\circ} \mathrm{C}, \geq 4 D A T+$ at least four different direct antiglobulin test results were positive among the five to six performed DATs

${ }^{a}$ Blood smears from six dogs could not be assessed due to strong agglutination, except for the presence of autoagglutination 


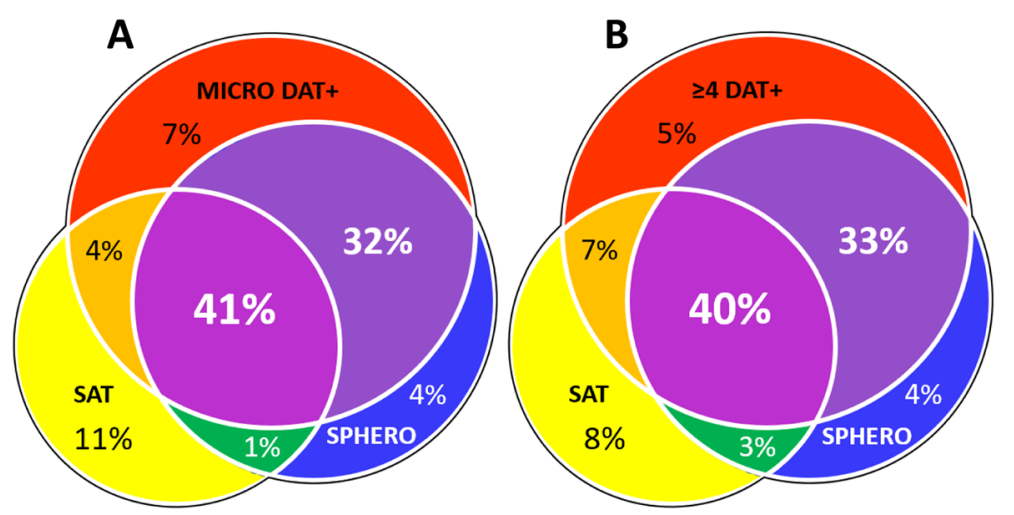

Fig. 1 Venn diagrams comparing agglutination, spherocytosis, and DAT+ results in 120 dogs suspected to have IMHA. Microtiter plate direct antiglobulin test (MICRO) results with goat anti-canine IgG, IgM, and $\mathrm{C} 3$ incubated at $22^{\circ} \mathrm{C}(\mathbf{A})$ and $\geq 4$ DAT+ results $(\mathbf{B})$. Six of 126 cases were excluded due to strong agglutination making the evaluation of the presence of spherocytosis impossible. MICRO DAT+: microtiter plate direct antiglobulin test method with goat anti-dog $\operatorname{lgG}, \operatorname{lgM}$, and $\mathrm{C} 3$ incubated at $22^{\circ} \mathrm{C} ; \mathrm{SAT}: 1: 1$ macroscopic saline agglutination test before washing; SPHERO: spherocytosis defined as $\geq 5$ spherocytes per 100 high power field

samples were still slightly agglutinated (Table 1). Therefore, despite originally agglutinating, analysis of DAT could be attempted in all dogs and in nearly all cases was interpretable (see below). Remarkably, DAT results were negative in ten of the agglutinating samples ( 20\%) (Fig. 1).

\section{Direct antiglobulin test (DAT) results} Laboratory microtiter plate (MICRO) DAT method MICRO DAT analysis using GAD at $22^{\circ} \mathrm{C}$, identified 67 of 126 samples as positive, with strongly to moderately and rarely weakly positive DAT titers (Table 2). In half of the MICRO GAD DAT+samples, a prozone effect was observed in moderately to strongly DAT+samples (titer $\geq 1: 256$ with MICRO GAD $22^{\circ} \mathrm{C}$, Table 2 and

Table 2 Direct antiglobulin test results compared to titers and prozone effect in dogs suspected to have IMHA

\begin{tabular}{lllll}
\hline MICRO DAT & Titer & $\begin{array}{l}\text { Frequency } \\
\mathbf{n}(\%)\end{array}$ & $\begin{array}{l}\text { Prozone } \\
\mathbf{n}(\%)\end{array}$ & $\begin{array}{l}\mathbf{2} \mathbf{4} \text { DAT+ } \\
\mathbf{n}(\%)\end{array}$ \\
\hline DAT- & $<1: 2$ & $56(44)$ & 0 & $3(4)$ \\
& $1: 2$ & $1(1)$ & 0 & $1(1)$ \\
DAT+ & $1: 4$ & $2(2)$ & 0 & $2(3)$ \\
& $1: 32$ & $2(20$ & 0 & $1(1)$ \\
& $1: 64$ & $1(1)$ & 0 & $1(1)$ \\
& $1: 256$ & $4(3)$ & $1(3)$ & $4(6)$ \\
& $1: 512$ & $6(5)$ & $3(9)$ & $5(7)$ \\
& $1: 1024$ & $6(5)$ & 0 & $5(7)$ \\
Total Dogs $\mathbf{n}$ & $1: 2048$ & $48(38)$ & $30(88)$ & $45(67)$ \\
\hline
\end{tabular}

Titers of 1:8 and 1:16 were not seen; $n$ : number of dogs

DAT direct antiglobulin test, MICRO DAT microtiter plate DAT with goat anti-dog $\operatorname{lgG}$, IgM, and $\mathrm{C} 3$ reagent incubated at $22^{\circ} \mathrm{C}, \geq 4 \mathrm{DAT}+$ at least four different DAT results were positive among the five to six performed DATs
Fig. 2A). Among the 59 MICRO GAD DAT- dogs, there were 18 samples that tested DA + by one $(n=15)$ and rarely more $(1 \mathrm{DAT}+=15,2 \mathrm{DAT}+=2,3 \mathrm{DAT}+=1)$ of the other DAT methods, and 16 of them were only weakly DAT+ (Table 3 ).

Performing the MICRO GAD DAT at $37^{\circ} \mathrm{C}$ and $4^{\circ} \mathrm{C}$ produced very similar results to those obtained at $22^{\circ} \mathrm{C}$ (Suppl Table S8). Likewise, replacement of GAD with the RAD antiglobulin and evaluation of the DAT at each temperature revealed similar results (Suppl Table S8).

\section{Immunochromatographic strip kit (STRIP KIT) DAT method}

Using the STRIP KIT DAT method with RAD antiglobulin at $22^{\circ} \mathrm{C}$ (Alvedia), 79 of 126 washed blood samples tested DAT+(Suppl Table S9 and Fig. 2B). This included seven samples showing a strong band at the origin (bottom) of the strip, indicative of autoagglutination. These samples were also agglutinating on slides (and two of them showed persistent agglutination), tested DAT+by other methods, and were considered strongly DAT+. Strongly positive $(4+$ and $3+)$ bands were seen with the STRIP KIT DAT in 30 dogs, with half of the remaining DAT + samples showing moderate $(2+)$ or weak $(1+)$ binding at the strip's test site, respectively (Suppl Table S9). All samples testing DAT+by MICRO GAD DAT were STRIP KIT DAT+, except for one, and were frequently also DAT+ by other DAT methods. In addition, 11 MICRO DAT- samples showed moderately $(n=3)$ to weakly $(n=8)$ positive band strength by STRIP KIT DAT (Suppl Table S9).

\section{Gel minitube kit (GEL KIT) DAT method}

Using unwashed blood with the GEL KIT DAT and $\mathrm{RAD}$ at $22^{\circ} \mathrm{C}$ (Alvedia), 74 of 126 samples were 


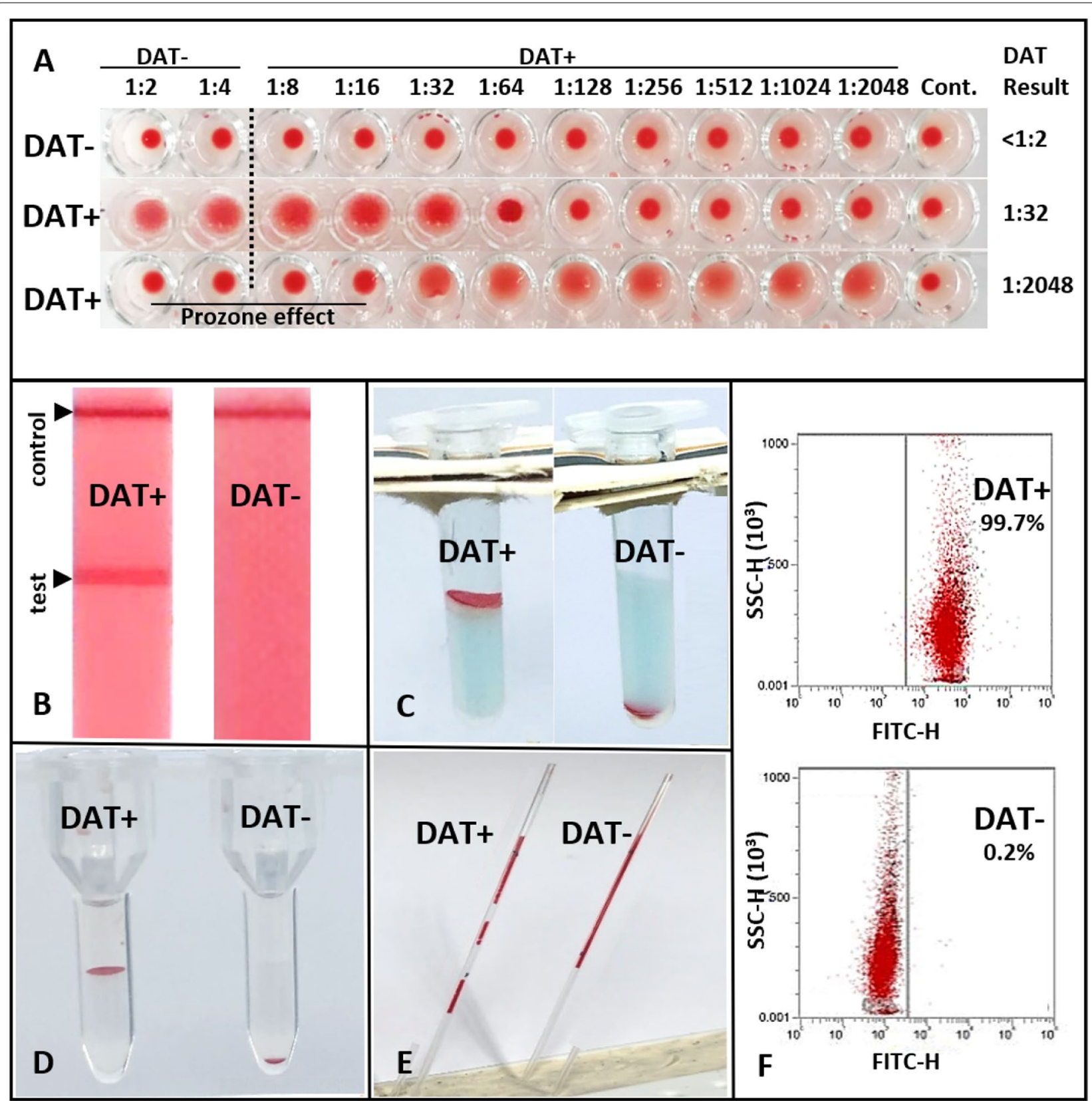

Fig. 2 Representative positive and negative direct antiglobulin test (DAT) results performed with different methods (A) microtiter plate; $\mathbf{B}$ in-clinic immunochromatographic strip kit; $\mathbf{C}$ in-clinic gel minitube kit; $\mathbf{D}$ neutral gel column card with added antiglobulin; $\mathbf{E}$ microcapillary tube; and $\mathbf{E}$ flow cytometry DAT \pm : direct antiglobulin test positive/negative result; Cont: control (saline but no antiglobulin added)

identified as DAT+. For all GEL KIT DAT+samples the RBCs accumulated at the top $(4+$ or $3+)$ allowing for easy test interpretation (Fig. 2C). However, a double population of RBCs at the top and bottom of gel minitubes was noted in 13 samples that were still graded as 4+(Suppl Table S9). All samples testing DAT+ by routine MICRO GAD DAT were DAT+ by the
GEL KIT DAT, except for three (those were, however, GEL LAB DAT+). All samples with $\geq 4$ DAT+results were also GEL KIT DAT+. Based upon the clinicopathological information received for 42 cases, only one of these dogs was transfused before testing. Washing the RBCs prior to performing the GEL KIT DAT produced similar results (Suppl Table S10). 
Table 3 Direct antiglobulin test results with five methods in dogs suspected to have IMHA

\begin{tabular}{lllllll}
\hline DAT Method & DAT- & 1 DAT+ & 2 DAT+ & 3 DAT+ & 4 DAT+ & 5 DAT+ \\
\hline MICRO & 35 & 0 & 1 & 6 & 13 & 53 \\
GEL KIT & 35 & 2 & 1 & 4 & 1 & 53 \\
GEL LAB & 35 & 7 & 0 & 5 & 53 & 53 \\
STRIP KIT & 35 & 6 & 0 & 5 & 13 & 53 \\
CAPIL & 35 & 0 & $\mathbf{2}$ & $\mathbf{7}$ & $\mathbf{1 4}$ & 53 \\
Total $(n=126)$ & $\mathbf{3 5}$ & $\mathbf{1 5}$ & $\mathbf{5 3}$
\end{tabular}

Results with MICRO DAT include all reagents and temperature; results of GEL LAB and CAPIL DAT with both reagents. Any positivity with one or more reagents or temperatures was considered DAT+

DAT Direct antiglobulin test, STRIP KIT in-clinic immunochromatographic strip kit, CAPIL microcapillary tube, MICRO microtiter plate, GEL KIT in-clinic gel minitube kit, GEL LAB neutral gel column card with added antiglobulin, FLOW was excluded because of lower number of samples tested

\section{Laboratory gel card (GEL LAB) DAT method with neutral gel column card}

Utilizing the GEL LAB DAT method with GAD antiglobulin and neutral gel column cards, 52 of 126 samples were DAT + . The degree of agglutination reaction was strong (4+or 3+, Fig. 2D) for 38 samples, and the DAT results were moderately $(2+)$ or weakly $(1+)$ positive for an additional four and ten samples, respectively. Except for five cases, all GEL LAB GAD DAT+samples were also positive with MICRO GAD DAT and most other DAT methods (except for two). In addition, 20 samples were GEL LAB DAT- but MICRO GAD DAT+(Suppl Table S9), and 17 of these 20 cases were DAT+ by all other DAT methods.

Replacing the GAD with the RAD antiglobulin reagent produced similar GEL LAB DAT results. The degree of agglutination was strong $(4+$ or $3+)$ for 42 samples, and moderately $(2+)$ or weakly $(1+)$ positive for seven and 12 samples, respectively. All GEL LAB RAD DAT+samples were also positive with MICRO GAD DAT except for seven weakly and four strongly GEL LAB DAT+samples (Suppl Table S9). Furthermore, 17 GEL LAB DAT- samples with RAD were MICRO GAD DAT+; and similarly, 14 of these 17 samples were DAT+ with all other DAT methods.

\section{Capillary (CAPIL) DAT method}

The capillary DAT utilizing a $30 \%$ washed RBC suspension and either the GAD or RAD reagent, revealed that 55 and 68 of 126 tested samples were DAT+, respectively (Suppl Table S9 and Fig. 2E). All CAPIL DAT+ samples were also positive by the MICRO GAD DAT (except for four and six cases for GAD and RAD, respectively) and by most other DAT methods $(\geq 4$ DAT+; except for one and five cases, respectively). A discordance of CAPIL DAT- and MICRO GAD DAT + was rarely observed with RAD (five cases) but more frequently when GAD was used as the antiglobulin (16 cases) in the CAPIL DAT method (Suppl Table S9).

\section{Laboratory flow cytometric (FLOW) DAT method}

The setup of the flow cytometric DAT method was initially hampered by interfering $\mathrm{RBC}$ agglutination leading to loss of RBCs and technical flow problems and thus was only included for the second half of the samples. Utilizing an anti-IgG specific antiglobulin reagent, the proportion of goat anti-dog FITC marked IgG antiglobulin-bound RBCs observed among the 69 flow cytometrically tested samples ranged from 1 to $100 \%$. With the established $<6 \%$ positive erythrocytes as unspecific background, based on our negative control dogs, and thus categorized as DAT-, 51 samples were FLOW DAT+. The amount of bound antiglobulin varied from 8 to $100 \%$ for DAT+ samples (Figs. 2F and 3). Of the 51 FLOW DAT+samples, 41 were also MICRO GAD DAT+. Two FLOW DAT- samples were in the group of $\geq 4 \mathrm{DAT}+$, and five FLOW DAT+samples were DAT- by all other methods (Suppl Table S9).

\section{Comparative analyses of immunohematological test results}

The Cohen k-values of all possible pairs of DAT methods and their confidence interval (CI) are represented for the 126 dogs suspected of having IMHA in Table 4 and Suppl Table S11. The Fleiss kappa value overall agreement was 0.73 (CI $0.70-0.75, p<0.001$ ) for all 126 samples for which a DAT was requested and 0.79 (CI $0.77-0.81, p<0.001$ ) when including the 28 samples from healthy control dogs, thus both reflect a "good agreement" [31]. In addition, the Cohen's $\kappa$-values were analyzed for all dogs including the 28 healthy control dogs in Suppl Table S12. The MICRO DAT with GAD at 


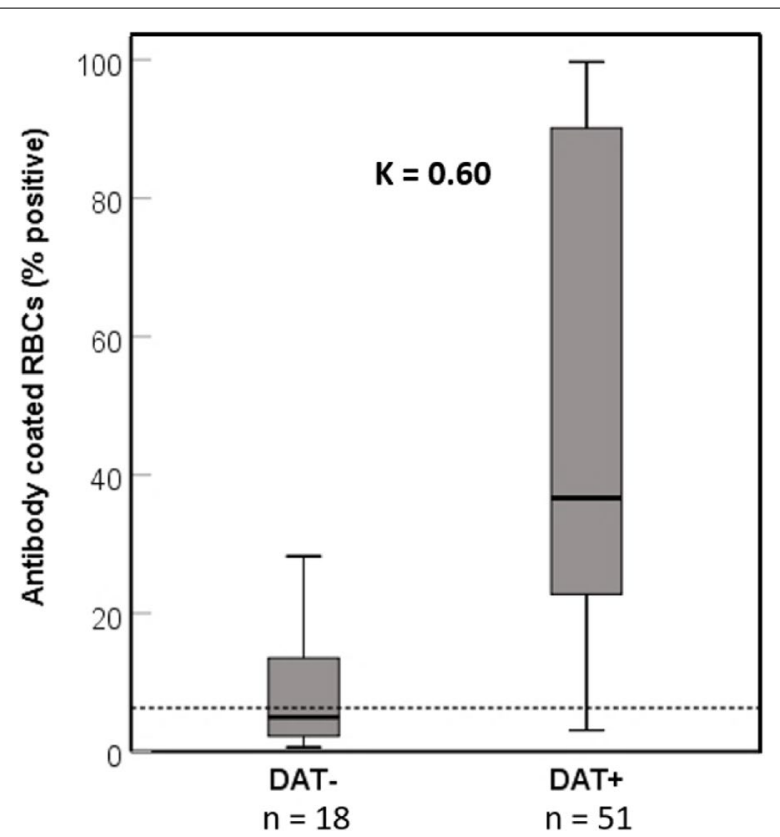

Fig. 3 Comparison of flow cytometric and microtiter plate DAT results in 69 dogs suspected to have IMHA. DAT: direct antiglobulin test. Red blood cells (RBCs) marked positive with FITC goat anti-dog IgG were expressed in percentages. The dotted line represents upper limit of normal (6\%; mean +2 SD of 13 control samples). Groups were compared by Cohen's kappa, $\mathrm{K}=.60$ (95\% Cl, .41-.80, $p<.001)$. Interpretation of $\mathrm{k}$ values: very good agreement ( $\geq .81)$; good agreement $(\geq 0.61)$; moderate agreement $(\geq .41)$; fair agreement ( $\geq$.21), as per Landis and Koch [30] and adapted by Brennan and Silman [31]; SD: standard deviation

$22^{\circ} \mathrm{C}$ results showed a very good agreement $(\kappa=0.81-$ 1.0) with each reagent and temperature. The results of CAPIL DAT with RAD also showed a very good agreement with MICRO DAT and with the GEL KIT DAT results. The CAPIL DAT with GAD, the STRIP KIT DAT, the FLOW DAT, and the GEL LAB DAT with GAD revealed good agreement when compared to every other DAT method ( $\kappa=0.61-0.8)$, while a comparison of the GEL LAB DAT with RAD showed a moderate agreement $(K=0.41-0.6)$ [32].

When comparing the results of the MICRO DAT with $\mathrm{GAD}$ at $22^{\circ} \mathrm{C}$ as a reference ("gold standard") method, all other DAT methods showed an analytical sensitivity $>90 \%$, except for the CAPIL DAT with GAD and the GEL LAB DAT with either antiglobulins. The analytical specificity of DAT results was also $>90 \%$, except for the STRIP KIT DAT, FLOW DAT, and GEL LAB DAT with RAD before washing (Table 5).

The Cohen $\mathrm{k}$-values, analytical specificity, and sensitivity of the different agglutination tests were modest with a k-value ranging from 0.39 before to 0.05 after washing (Suppl Table S13). The sensitivity for agglutination ranged from $6 \%$ before washing to $57 \%$ after washing, and the specificity from $83 \%$ before washing to $100 \%$ after washing when compared to the MICRO GAD DAT at $22^{\circ} \mathrm{C}$. The analytical sensitivity for spherocytosis was $87 \%$ and the specificity $93 \%$.

\section{Follow-up test results for $12 \mathrm{DAT}+$ dogs}

All 67 dogs with DAT+ results were followed up by contacting the submitting clinic (including reminders) with the recommendation that they retest during treatment. In a total of 42 responses, $13 \mathrm{DAT}+$ dogs were reported to have IMHA and died or were euthanized. The other 29 $\mathrm{DAT}+$ dogs were also clinically diagnosed with IMHA, but were either clinically stable $(n=16)$ or recovered $(n=13)$.

Despite the encouragement for retesting, samples for immunohematological reassessment were only received from 12 of the DAT+dogs (twice for three dogs) at different time intervals from diagnosis. Seven still showed clinical signs and five were clinically assessed as stable at the time of testing. After 1-2 weeks, the tested dogs were all still anemic $(n=4)$. From week 4 post-treatment, all dogs retested were no longer anemic, except for one, which was still anemic at week 24 . Only three dogs were DAT-, while all others $(n=9)$ remained DAT+when retested between 1 and 24 weeks post-diagnosis. Those dogs which became DAT- also resolved their spherocytosis and autoagglutination.

Furthermore, three DAT + dogs tested at weeks 2, 8, and 12 post-treatment were reassessed at weeks 19,20 , and 24 , respectively. Two of the samples were still DAT + and had spherocytosis and hemolysis, and one was still macroscopically agglutinating at first retesting (Table 6). At the second retesting, two dogs were DAT-, and had no spherocytosis, and the other still was anemic and had a $\mathrm{DAT}+$ result. That dog was euthanized shortly thereafter.

\section{Discussion}

While reports of IMHA in dogs are widespread, the diagnostic approach of the disease remains challenging and controversial, and rarely leads to a definitive diagnosis $[2,4,25,41]$. IMHA is driven by antibody-mediated destruction of RBCs, however no reference (gold standard) diagnostic DAT method has been established so far, and no preferred antiglobulin has been identified yet [3, $6,8,25,42]$. To determine the variability and accuracy of currently used methods, we undertook a prospective comparative study of immunohematological tests for canine IMHA. In this large cohort of 126 dogs suspected of having IMHA and 28 healthy control dogs about half of the samples tested had evidence of antibody-coated erythrocytes regardless of what immunohematological test was used. Moreover, we obtained similar and consistent results using various DAT methods. Our results 
Table 4 Comparison of Cohen's kappa ( $\mathrm{k}$ ) values of six direct antiglobulin test (DAT) results from 126 dogs suspected to have IMHA

\begin{tabular}{|c|c|c|c|c|c|c|c|c|}
\hline \multirow{2}{*}{$\begin{array}{c}\text { DAT } \\
\text { Method }\end{array}$} & \multirow{2}{*}{ Antiglobulin } & \multicolumn{2}{|c|}{ CAPIL } & \multirow{2}{*}{$\begin{array}{c}\text { MICRO } \\
\text { GAD } \\
22^{\circ} \mathrm{C}\end{array}$} & \multirow{2}{*}{$\begin{array}{l}\text { GEL } \\
\text { KIT } \\
\text { RAD } \\
22^{\circ} \mathrm{C}\end{array}$} & \multicolumn{2}{|c|}{ GEL LAB } & \multirow{2}{*}{$\begin{array}{l}\text { FLOW } \\
\text { GAD* }^{*} \\
22^{\circ} \mathrm{C} \\
\end{array}$} \\
\hline & & $\begin{array}{l}\text { GAD } \\
22^{\circ} \mathrm{C}\end{array}$ & $\begin{array}{l}\text { RAD } \\
22^{\circ} \mathrm{C}\end{array}$ & & & $\begin{array}{l}\text { GAD } \\
22^{\circ} \mathrm{C}\end{array}$ & $\begin{array}{l}\text { RAD } \\
22^{\circ} \mathrm{C}\end{array}$ & \\
\hline STRIP & $\operatorname{RAD} 22^{\circ} \mathrm{C}$ & .56 & .72 & .77 & .75 & .52 & .46 & .66 \\
\hline \multirow{2}{*}{ CAPIL } & GAD $22^{\circ} \mathrm{C}$ & 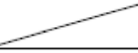 & .76 & .68 & .70 & .72 & .64 & .46 \\
\hline & $\operatorname{RAD} 22^{\circ} \mathrm{C}$ & 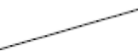 & & .82 & .80 & .68 & .60 & .60 \\
\hline MICRO & $\mathrm{GAD} 22^{\circ} \mathrm{C}$ & $=$ & & $\Omega$ & .79 & .60 & .55 & .60 \\
\hline GEL KIT & $\operatorname{RAD} 22^{\circ} \mathrm{C}$ & 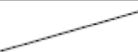 & & $>$ & $>$ & .66 & .57 & .66 \\
\hline \multirow{2}{*}{ GEL LAB } & $\mathrm{GAD} 22^{\circ} \mathrm{C}$ & $\pi$ & & 2 & 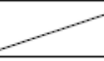 & 7 & .69 & .39 \\
\hline & $\operatorname{RAD} 22^{\circ} \mathrm{C}$ & & & & & & 7 & .37 \\
\hline
\end{tabular}

DAT: direct antiglobulin test, DAT methods: STRIP KIT: in-clinic immunochromatographic strip kit; FLOW: flow cytometry ( $n=69)$; CAPIL: microcapillary tube; MICRO: microtiter plate; GEL KIT: in-clinic gel minitube kit; GEL LAB: neutral gel column card with added antiglobulin Reagents: GAD: goat anti-dog lgG, IgM, and C3; RAD: rabbit anti-dog IgG, IgM, and C3, GAD*: FITC-marked goat anti-dog Ig $(\mathrm{H}+\mathrm{L})$ Interpretation of $\boldsymbol{\kappa}$ values: Groups were compared by Cohen's kappa, $\mathrm{K}=.37$ to .82 , $p<.001$ Green: very good agreement $(\geq .81)$; yellow: good agreement $(\geq .61)$; blue: moderate agreement $(\geq .41)$; white: fair agreement $(\geq .21)$, as per Landis and Koch [32] and adapted by Brennan and Silman [33]

Table 5 Results of six DAT methods compared to microtiter plate DAT in 126 dogs suspected to have IMHA

\begin{tabular}{|c|c|c|c|c|c|}
\hline $\begin{array}{l}\text { DAT } \\
\text { Method }\end{array}$ & Reagent & $\begin{array}{c}\text { Incubation } \\
\left({ }^{\circ} \mathrm{C}\right)\end{array}$ & $\begin{array}{c}\text { Washing } \\
\text { With Saline }\end{array}$ & $\begin{array}{c}\text { Analytical } \\
\text { Sensitivity } \\
(\%)\end{array}$ & $\begin{array}{c}\text { Analytical } \\
\text { Specificity } \\
(\%)\end{array}$ \\
\hline STRIP KIT & RAD & 22 & Yes & 98.5 & 78.0 \\
\hline FLOW & GAD* & 22 & Yes & 95.3 & 61.5 \\
\hline \multirow{2}{*}{ CAPIL } & GAD & 22 & Yes & 76.1 & 93.2 \\
\hline & RAD & 22 & Yes & 92.5 & 89.8 \\
\hline \multirow{6}{*}{ MICRO } & GAD & 37 & Yes & 98.5 & 96.6 \\
\hline & GAD & 22 & NA & NA & NA \\
\hline & GAD & 4 & Yes & 97.0 & 96.6 \\
\hline & RAD & 37 & Yes & 95.5 & 96.6 \\
\hline & RAD & 22 & Yes & 95.5 & 91.5 \\
\hline & RAD & 4 & Yes & 92.5 & 93.2 \\
\hline \multirow{2}{*}{ GEL KIT } & RAD & 22 & No & 95.5 & 83.1 \\
\hline & RAD & 22 & Yes & 94.0 & 88.1 \\
\hline \multirow{4}{*}{ GEL LAB } & GAD & 22 & No & 100 & 79.7 \\
\hline & GAD & 22 & Yes & 70.1 & 91.5 \\
\hline & RAD & 22 & No & 100 & 42.4 \\
\hline & RAD & 22 & Yes & 74.6 & 81.4 \\
\hline
\end{tabular}

DAT: Direct antiglobulin test

DAT methods: STRIP: in-clinic immunochromatographic strip kit; FLOW: flow cytometry ( $n=69)$; CAPIL: microcapillary tube; MICRO: microtiter plate; GEL KIT: in-clinic gel minitube kit; GEL LAB: neutral gel column card with added antiglobulin

Reagents: GAD: goat anti-dog IgG, IgM, and C3; RAD: rabbit anti-dog IgG, IgM, and C3, GAD*: FITC-marked goat anti-dog IgG $(\mathrm{H}+\mathrm{L})$ Interpretation: $>90 \%$ (bold) represent excellent analytical sensitivity and specificity values NA: not applicable, because everything was compared to MICRO GAD $22^{\circ} \mathrm{C}$ 


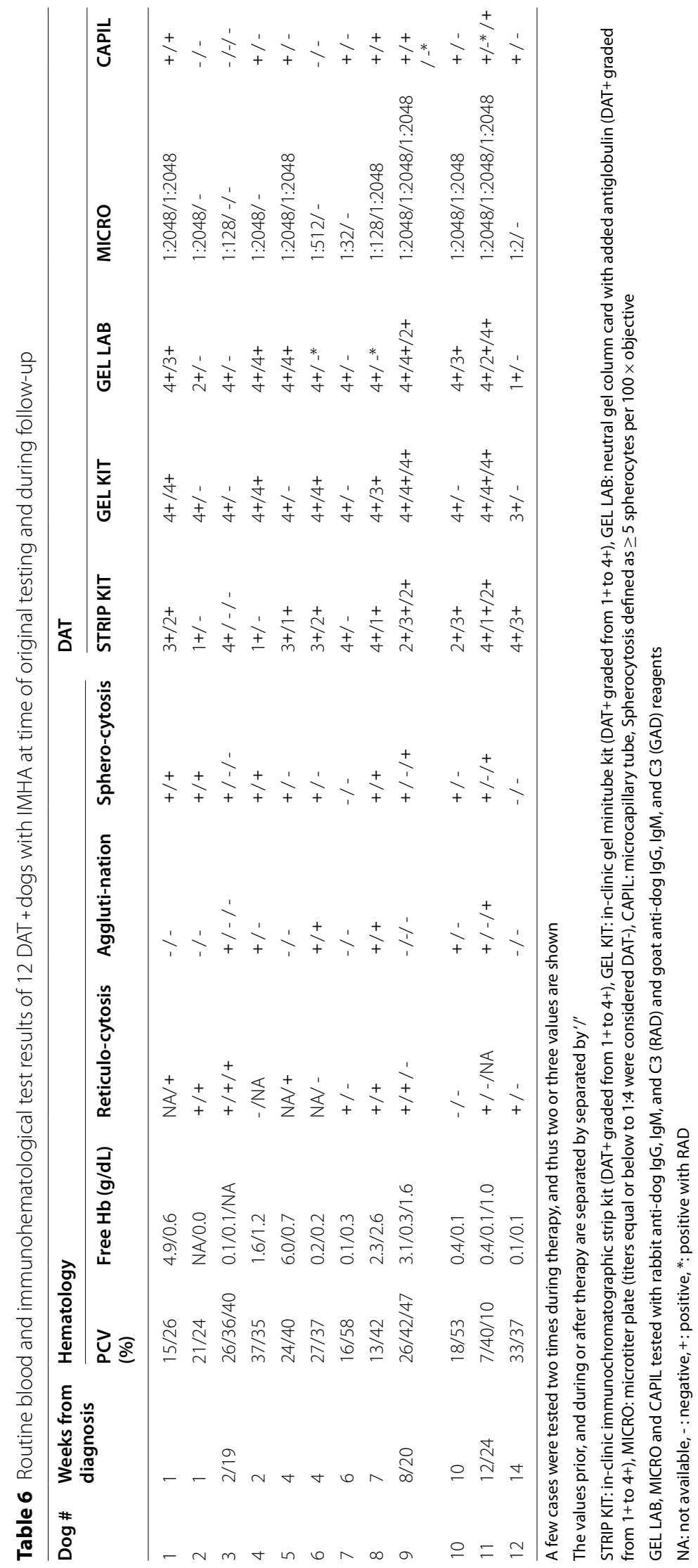


underscore the usefulness of various DAT methods in the diagnosis of IMHA.

\section{Signalment and routine blood test results of DAT + and DAT- dogs}

With respect to signalment, middle-aged and female dogs were overrepresented as reported in prior retrospective surveys [43-45], but there was neither an apparent breed predilection among dogs for which a DAT was requested nor for those with DAT+results. In past studies the Cocker Spaniel was overrepresented [24, 43, 45-48], and a recent survey from the United Kingdom suggested a few other breeds with higher odd ratios for non-regenerative IMHA [19].

Among the samples tested in this study, dogs with a positive DAT result had more frequent and more severe anemia (Hct $<18 \%)$ as was previously described $[44,46]$, and the anemia was more likely regenerative in contrast to those in the DAT- group $[19,25,43]$. While there is no published study to directly compare our observations to, it is likely that some DAT- dogs had non-hemolytic anemias. In this study, both DAT+and DAT- samples appeared hemolyzed, but the degree of hemolysis was higher in DAT+ samples, which may represent an artifact caused by in vitro lysis during shipment and storage (up to 5 days from collection). It is therefore not surprising to find discrepancies between $\mathrm{Hb}, \mathrm{PCV}$, and Hct values observed in the study reported here and in clinical practice [49]. When serum chemistry was available, we found hyperbilirubinemia more frequently in DAT+dogs as expected in dogs with hemolytic anemia [46].

Similar to prior surveys of dogs with IMHA [25, 43, 44], not all DAT+dogs showed evidence of erythroid regeneration. This may be due to pre-regenerative anemia (first 2-3 days of anemia), mild anemia, or inflammatory and necrotic processes associated with IMHA in dogs, leading to inhibition of adequate erythroid regeneration in the bone marrow [19, 50, 51]. Varied erythroid responses and bone marrow cytological and histological abnormalities have been documented in dogs with IMHA, but time-course was not evaluated $[19,51,52]$.

\section{Spherocytosis}

In the present study, $\geq 5$ spherocytes per high power microscopic field $[6,24,25]$ were primarily seen in $\mathrm{DAT}+$ dogs, with only four exceptions. Low numbers of spherocytes may be observed in many disease processes $[1,53]$. In addition, hereditary spherocytosis, although rare in dogs, is associated with severe spherocytosis [54, 55]. Moreover, spherocytes may also represent artifacts and are commonly seen in thicker areas of blood smears [56]. Thus, in dogs with spherocytosis, DAT testing can play an important role in the diagnostic process, with DAT- results helping to rule out IMHA. In addition, although spherocytosis provides strong evidence for IMHA, DAT+dogs without spherocytosis are not uncommon, representing 5 to $25 \%$ in previous studies [56], which correlates well with our finding of $13 \%$ of the DAT+ animals being negative for spherocytosis.

\section{Autoagglutination and SAT}

There is considerable controversy regarding the best means to assess and interpret autoagglutination in dogs, and when noted, whether a DAT could and should still be performed $[3,4,6,20]$. As expected, more DAT+ samples agglutinated than did DAT- samples in the present study. In fact, in many blood samples agglutination occurred in the EDTA tube, and persisted after 1:1 and 1:4 saline dilution. Previous studies noted that $42-87 \%$ of samples from dogs clinically suspected to have IMHA showed positive slide/saline agglutination [43, 44, 48]. In the current study, $48 \%$ showed positive SAT results, but one fifth of macroscopically agglutinating samples were DAT-. The proportion of agglutinating DAT- samples is also similar to prior smaller studies [3, 24, 46]. Thus, macroscopic agglutination in the tube and/or a positive SAT result do not document antibody-bound erythrocytes and are not truly diagnostic for IMHA.

While the SAT is recommended by the ACVIM consensus statement [6], we had questioned its value in the past $[1,5]$. A recent study also suggested that a SAT using a 1:1 and 1:4 blood to phosphate-buffered saline dilution with microscopic evaluation was not a useful diagnostic test for IMHA in dogs [20]. However, the diagnostic accuracy of a 1:49 dilution SAT with microscopic evaluation was much higher, which was attributed to a reduction in non-specific agglutination as happens with saline washing of RBCs [20]. We have previously advocated three saline washes to remove all potentially interfering plasma components and EDTA, as it is standard in human immunohematology [8]. Indeed only four of 48 samples still revealed minor macroscopic agglutination after washing in the current study. These findings suggest that three saline washes should be seen as the standard procedure for immunodiagnostics in dogs [57]. While it is possible that unknown low affinity autoantibodies could dissociate from the RBC surface in the presence of saline [58], such low-affinity antibodies are rarely documented in humans and have not been identified in dogs. Finally, results of agglutination after washing with saline are rarely reported in dogs $[20,25,43,59]$.

Rouleaux formation did not appear to cause the observed macroscopic autoagglutination, as a combination of agglutination and rouleaux were noted in only a few $(8 \%)$ cases in the present study. It should also be 
noted that rouleaux only cause microscopic and not macroscopic agglutination [56].

\section{Direct antiglobulin test (DAT)}

As there is skepticism concerning the value of the DAT in canine medicine, due in part to the lack of a reference (gold standard) for any DAT technique and antiglobulin selection $[25,60,61]$, we undertook this extensive comparative analysis of multiple techniques currently in use in clinics and laboratories. Our results demonstrate encouragingly good concordance between all DAT methods applied. Because making a definitive diagnosis of IMHA is challenging and immunodiagnostic methods applied vary and are not standardized, accurate assessments of specificity and sensitivity of the various tests are difficult to carry out in the field. Thus, our comparative study of six DAT methods in a controlled laboratory setting provides reassuring evidence that the results of all DAT methods are in overall good agreement, as discussed further below.

The microtiter plate (MICRO) DAT method had been extensively used in human and veterinary laboratories $[3,8,13-15,42,59,62]$. We applied it here as the reference (gold standard) method and demonstrated very good analytical sensitivity and specificity (ranging between 92-99\%), even at different temperatures and with two different polyclonal reagents. Thus, the simple room temperature condition appears to be sufficient. Prior studies $[18,21,59,63]$ show similar specificity, from 95 to $100 \%$, but lower sensitivity, between 53 and $82 \%$, for IMHA. However, sensitivity in those studies were based on a clinical diagnosis of IMHA, and not necessarily on documentation of immune destruction of erythrocytes.

The results of the in-clinic immunochromatographic strip kit (STRIP KIT) DAT test with RAD at $22^{\circ} \mathrm{C}$ in the present study concurs with a prior smaller DAT study regarding analytic sensitivity, but appeared to be slightly less specific [3]. The STRIP KIT test is also used for $D E A$ 1 blood typing, where its specificity is described to be very high [64]. A limitation of this method appears to be the rare occurrence $(5 \%)$ of autoagglutination at the bottom of the strip, thereby impeding the normal flow of erythrocytes past the test and control bands. This occurred in 7 of our 154 samples which all showed macroscopic agglutination. Moreover, the interpretation of weakly positive DAT reactions can be problematic in clinical practice, but occurred less commonly than in our prior limited study [3].

While washing the cells is recommended prior to most DAT methods, this study demonstrates that the in-clinic gel minitube kit (GEL KIT) DAT results with and without washing are almost identical. Thus, samples for the gel test do not necessarily need to be washed, as long as the negative gel control microtube is negative. Due to its recent introduction, this method has never before been studied in comparison to other DAT techniques and has only been compared to SAT in one previous study [20]. Notably, our results identify the GEL KIT as the quickest and most reliable in-clinic DAT, suggesting that it represents an invaluable tool for differential diagnosis of IMHA in clinical practice.

The neutral gel column card (GEL LAB) DAT is often used as a standard test in human medicine, where its ease of result interpretation and high sensitivity have been praised $[15,65]$. The first dog-specific commercial laboratory gel DAT was tested and recommended in two previous publications [3, 62]. Studies comparing neutral gel columns to microtiter plates reported moderate to very good agreement $[32,33])$. One laboratory [62] reported agreement slightly lower than the values reported in the current study. Another study [3] describes a very good agreement with the MICRO DAT method. Since that original canine gel column product was discontinued shortly after its introduction, we used neutral gel cards and added a canine-specific antiglobulin reagent in the reaction chamber. This assay was easy to perform and produced very similar results with either GAD or RAD, comparable to the other DAT methods.

Similar to our prior smaller study using the microcapillary tube (CAPIL) DAT [3], 85\% MICRO DAT+samples were CAPIL DAT+, with an analytical sensitivity and specificity above $90 \%$ achieved. The CAPIL DAT is frequently performed in human medicine, particularly in blood banking laboratories [13] to assess blood type incompatibilities. Notably, the simplicity of this technique could make it attractive as an in-clinic and laboratory screening test, when more advanced DAT methods and DAT kits are not available.

While our study data for flow cytometry (FLOW) DAT only tested 69 of 123 dogs, the results were similar to other DAT results. However, there was a somewhat larger proportion of discordant DAT+ and DAT- results when compared with other DAT methods, revealing a very good sensitivity (95\%), but a low specificity $(62 \%)$. The percentage of positive also lacked any specific association with the degree of anemia and hemolysis. Prior studies of FLOW DAT are limited, but reveal sensitivities and specificities ranging from 74 to $100 \%$, when compared to a clinical diagnosis of IMHA [18, 21, 60, 63]. These variations may be related to different standard methods used. Prior limited studies with FLOW DAT suggested that the number of antibody positive RBCs is a better parameter than the degree of positivity, assuming proper gating of 
positive and negative cells $[18,63]$. The background level of up to $6 \%$ (mean $+2 \mathrm{SD}$ ) positive RBCs set in our study is similar to that of others [60]. Because addition of antiglobulin to sub-agglutinating antibody-coated RBCs promote agglutination, finding the correct balance for antiglobulin binding without inducing agglutination is tricky and requires constant monitoring during FLOW DAT performance. As flow cytometry of erythrocytes is laborious, requires an expensive tool, and proves difficult to perform due to the tendency of erythrocytic agglutination, and FLOW DAT results do not appear to be superior to those with other DAT methods used in this study, it is unlikely that this technique will replace other classical DAT methods under most settings $[66,67]$.

We used two well-known polyvalent antisera against IgG, IgM, and C3 generated in either goat or rabbit, and achieved very similar results by the MICRO, CAPIL, and GEL LAB DAT methods in this study. However, for FLOW DAT, the polyclonal antiglobulins appeared to produce nonspecific irreproducible results during setup, prompting us to use an IgG-specific antiglobulin reagent. A previous study reported that, in comparison to IgG alone, the use of IgG and IgM together dropped the specificity from 88 to $74 \%$, while the sensitivity stayed at $88 \%$ [60]. Those findings compare well with the data of this study.

The most common antiglobulin reagent and method reported in the literature is the microtiter plate DAT assay with $\operatorname{GAD}[3,11,59]$. The sensitivity of this reagent ranged from 61 to $95 \%$ in the literature, and the specificity ranged between 85 and $100 \%[3,11,59]$. While the difference between antiglobulin reagents used for MICRO DAT are negligible, in two other DAT assays (CAPIL and GEL LAB), RAD seemed to be more sensitive and GAD seemed to be more specific. The overall $\mathrm{k}$ values in this study should encourage the use of RAD for CAPIL and GAD for GEL LAB.

Our DAT+results with the anti-DEA 4 and anti-Dal antibodies and DAT- results with anti-DEA 5 in blood samples of control dogs with a DEA 4+, DEA 5-, and Dal+blood type support the specificity of these DAT techniques and antisera to detect RBCs coated with antibodies. The inclusion of DAT+and DAT- controls is important, and was also used in our prior DAT study [3], but it is rarely reported anywhere else. We realize that in vitro agglutination and binding reactions to alloantibodies may differ from that induced by autoantibodies in IMHA, but reagents consisting of canine autoantibodies are not available.

The main concern in IMHA are warm antibodies which are examined at body or room temperature. As the DAT results of this study were very similar if performed at $22^{\circ} \mathrm{C}$ or $37^{\circ} \mathrm{C}$, it appears sufficient to set up the simpler room temperature incubation. Cold-antibody-mediated IMHA is extremely rare in humans and has not been documented in dogs. Thus, DAT at $4^{\circ} \mathrm{C}$ is rarely performed in clinical practice. However, cold agglutinins may cause agglutination in extremities and thereby may result in ear, fingertip, nail, and tail necrosis $[8,68]$.

The MICRO DAT and FLOW techniques allow for quantitation of DAT results, while all other DAT methods give only semiquantitative results. Serial dilution of the antiglobulin reagent is recommended to overcome a prozone effect caused by an excess of antiglobulin concentration. And if serial dilutions are not performed samples could be falsely labelled DAT-. Notably a prozone effect at lower dilutions (Fig. 2A) was seen in one fifth of the samples tested here. However, we observed a prozone effect only in those samples with high antiglobulin titers in the MICRO DAT, using either antiglobulin. While many publications on DAT refer to the prozone effect, there are few studies in dogs documenting the degree and frequency of the prozone effect in canine blood samples [62]. Interestingly, samples with an apparent prozone effect always produced DAT+ results with the other DAT methods which use fixed concentrations of antiglobulin. This finding is in concordance with our earlier report [3] as well as those in human medicine [62]. Therefore, these DAT methods with fixed antiglobulin quantities simplify the testing process and still permit a semi-quantitative assessment. Indeed, it was encouraging to find in this comparative study that the two in-clinic DAT techniques produced similar results to the more elaborated laboratory DAT methods.

To date, there is no clinical evidence that a higher MICRO DAT titer, number of DAT+RBCs by FLOW or higher scores of the semiquantitative DAT methods are associated with a more severe clinical presentation, worse prognosis, and/or worse response of dogs with IMHA to treatment. In the present study, there was only a fair correlation between degree of DAT positivity among tests. Likewise, the degree of DAT positivity did not appear to be associated with the degree of anemia, erythroid regeneration, agglutination, or spherocytosis in this study. However, titers and semiquantitative scores may be helpful in monitoring disease and response to treatment.

\section{Follow-up test results of DAT+ cases}

There is a paucity of data on dogs with IMHA during and after treatment $[3,24,69]$, with most information relating to complications and survival after varied treatments of IMHA and rarely including any (immuno-) hematological test results [3, 24, 46, 69]. It is possible that veterinarians assume that DAT results will turn immediately negative after starting immunosuppressive treatment, even if dogs remain anemic and hemolysis 
is unresolved. Moreover, many clinicians assume that blood transfusions will cause an immediate positive DAT result [6], despite the fact that dogs have no clinically important naturally occurring alloantibodies [70]. Finally, a positive DAT is rarely if ever observed in previously transfused dogs, even in those with acute hemolytic transfusion reactions [71].

Based upon experience in human medicine and our prior experience, dogs with IMHA continue to be $\mathrm{DAT}+$ until the antibody-mediated hemolysis resolves. As in our prior studies [3, 24], nine of $12 \mathrm{DAT}+$ dogs, that we were able to follow by testing, remained $\mathrm{DAT}+$ for weeks. Among the $12 \mathrm{DAT}+$ dogs monitored, 10 were no longer anemic when tested and in most cases their macroscopic agglutination and spherocytosis had resolved suggesting clinical improvement (was also the assessment by attending clinicians) while in part still being DAT+. Moreover, the 42 attending clinicians who responded reported that $70 \%$ of the DAT+ dogs with IMHA recovered or were clinically stable, while $30 \%$ of them died or were euthanized. These findings concur with prior outcome surveys [19, 72]. Thus, while the results of the follow-up data are sparse in this as well as in prior studies [3, 24, 25, 44, 69], they support the value of monitoring dogs with IMHA during and after treatment. Thus, the authors encourage clinicians to not only monitor the degree of anemia, but to also follow the erythroid regenerative response, autoagglutination, spherocytosis, and DAT when treating dogs with IMHA.

\section{Study limitations}

Samples were shipped to a large commercial laboratory and stored refrigerated for 2-5 days before analysis. Although this delay may have introduced artifacts, it reflects the clinical reality. Interestingly, agglutination, spherocytosis, and DAT results could still be well assessed, despite the delays. While fresh samples are certainly preferred for any morphological, immunological, and functional RBC studies, our study suggests that delays in testing of samples kept refrigerated do not hamper or preclude receiving meaningful positive immunohematological results. While some investigators had claimed that the DAT could only be done on fresh samples [16], our previous study [3] also indicated that the DAT results remain meaningful for blood that had been stored refrigerated for days. We have clinical follow-up information on only 42 of 67 DAT+dogs, and they were clinically assessed to have IMHA by the attending clinician. In addition, we have supportive routine and immunohematological results for those as well as all other DAT+dogs. As in other studies, we do not definitively know if any of the 126 dogs had or did not have IMHA. Finally, we did not investigate whether it was a primary (non-associative) or secondary (associative) IMHA.

The immunodiagnostic techniques were specifically established in one major veterinary diagnostic laboratory setting and performed each time by the same individual under essentially identical conditions. While this meant that the operator was not blinded to the other test results, every effort was made to objectively analyze each test result; and many results were and could be captured by photography for later review.

\section{Conclusions}

In this large cohort of 126 dogs suspected of having IMHA based on clinical data, CBC, and chemistry panel and 28 healthy control dogs, we obtained similar and consistent results using various DAT methods. There was also a good correlation between DAT and marked spherocytosis, but only fair agreement between DAT and agglutination, including SAT. The DAT methods, were both analytically more sensitive and more specific, detecting additional dogs with antibody-coated erythrocytes and excluding nonspecific agglutination. Furthermore, macroscopic autoagglutination only rarely interfered with interpretation of DAT results. Finally, DAT + dogs revealed DAT + results that persisted for weeks. This suggests that DAT might be useful in monitoring dogs receiving treatment for IMHA.This prospective study is the most comprehensive clinical comparative investigation of DAT methods to date, and showed excellent correlations between DAT methods. We hope that our findings will restore veterinary clinicians' confidence in DAT methods and results.

\section{Abbreviations}

ACVIM: American College of Veterinary Internal Medicine; BSA: Bovine serum albumin; CAPIL: Microcapillary tube; CAPIL GAD: Microcapillary tube with goat anti-dog lgG, IgM, and C3 antiglobulin; CAPIL RAD: With rabbit anti-dog IgG, IgM, and C3 antiglobulin; C3: Complement component 3; CBC: Complete blood count; Cl: Confidence interval; DAT+/DAT-: Direct antiglobulin test (with positive/negative result); DEA: Dog erythrocyte antigen; EDTA: Ethylenediaminetetraacetic acid; FITC: Fluorescein isothiocyanate; FLOW: Flow cytometry;

FITC-GAD: FITC-marked goat anti-dog IgG (heavy and light chains); GAD: Goat anti-dog IgG, IgM, and C3; GEL KIT: In-clinic gel minitube kit; GEL LAB: Neutral gel column card with added antiglobulin; GEL LAB GAD: Neutral gel column card with goat anti-dog lgG, IgM, and C3 antiglobulin; GEL LAB RAD: Neutral gel column card with rabbit anti-dog lgG, IgM, and C3 antiglobulin; Hct: Calculated hematocrit by hematology analyzer or $3 \times \mathrm{Hb}$ concentration in $\mathrm{g} / \mathrm{dL} ; \mathrm{Hb}$ : Hemoglobin; hpf: High-power field; h: Hour(s); IAT: Indirect antiglobulin test; IgG and IgM: Immunoglobulin G and M; IMHA: Immune-mediated hemolytic anemia; MFI: Mean fluorescence intensity; MICRO: Microtiter plate; MICRO GAD: Microtiter plate with goat anti-dog lgG, IgM, and C3 antiglobulin; MICRO RAD: Microtiter plate with rabbit anti-dog lgG, IgM, and $C 3$ antiglobulin; min: Minute(s); PBS: Phosphate-buffered saline; PCV: Packed cell volume; pRBCs: Packed red blood cells; RAD: Rabbit anti-dog lgG, IgM, and C3; RBC(s): Red blood cell(s); SAT: Saline agglutination test; SD/SE: Standard deviation/error of the mean; STRIP KIT: In-clinic immunochromatographic strip kit. 


\section{Supplementary Information}

The online version contains supplementary material available at https://doi. org/10.1186/s40575-021-00107-0.

Additional file 1: Supplementary Table S1. Test conditions for six direct antiglobulin test (DAT) methods. Supplementary Table S2. Demographic data of 126 dogs suspected to have immune-mediated hemolytic anemia (IMHA) and 28 healthy control dogs and with DAT+ results by microtiter. Plate direct antiglobulin test (DAT) with using goat anti-dog lgG, lgM, and $\mathrm{C} 3$ at $22^{\circ} \mathrm{C}$. Breed (A), age (B), and gender (C) distribution. Supplementary Tables S3. Demographic data of 126 dogs suspected to have immune-mediated hemolytic anemia (IMHA) with positive and negative direct antiglobulin test (DAT) results. Breed (A), age (B), and gender (C) distribution. Supplementary Table S4. In vitro-induced positive direct antiglobulin test (DAT) results in samples from eight healthy dogs with DEA 4+, DEA 5- and Dal+ blood type by adding anti-DEA 4, anti-DEA 5, and anti-Dal antisera. Supplementary Table $\mathbf{S 5}$. Anemia, reticulocytosis, hyperbilirubinemia, and hemolyzed plasma compared to DAT results in dogs suspected to have IMHA. Supplementary Table S6. Degree of anemia (total hemoglobin [Hb], packed cell volume [PCV], and hematocrit $[\mathrm{Hct}])$ in relation to direct antiglobulin test (DAT) results in dogs suspected to have immune-mediated hemolytic anemia. Supplementary Table S7. Correlation between polychromasia and reticulocytosis of samples from 80 dogs suspected to have immune-mediated hemolytic anemia and MICRO GAD direct antiglobulin test. Results from DAT+ dogs in brackets. Supplementary Table S8. Microtiter plate direct antiglobulin test (DAT) results with two antiglobulins (goat anti-dog; GAD and rabbit anti-dog; RAD) and at three different temperatures $\left(22,37\right.$ and $\left.4^{\circ} \mathrm{C}\right)$ for 126 dogs suspected to have immune-mediated hemolytic anemia. Supplementary Table S9. Comparison of six different direct antiglobulin tests (DAT) with one to two different antiglobulin reagent. Supplementary Table S10. Minitube gel kit direct antiglobulin test (GEL KIT DAT) in dogs suspected to have immune-mediated hemolytic anemia with and without prior $3 x$ washing of red blood cells. Supplementary Table S11. Comparison of six direct antiglobulin test (DAT) results in 126 dogs suspected to have immune-mediated hemolytic anemia (IMHA). The Cohen's kappa (K) values and a two-sided confidence intervals (95\%) is reported for each pair of methods used. Supplementary Table S12. Comparison of six direct antiglobulin test (DAT) results in 126 dogs suspected to have immunemediated hemolytic anemia (IMHA) and 28 healthy dogs. The Cohen's kappa ( $\mathrm{K}$ ) values and two-sided confidence intervals (95\%) are reported for each pair of methods used. Supplement Table S13. Cohen's kappa (K) value of the spherocytosis, and macroscopic in tube agglutination and macroscopic saline agglutination test (SAT) results at two dilutions compared to the microtiter plate DAT method with goat-anti dog lgG, $\lg \mathrm{M}$, and $\mathrm{C} 3$ at $22^{\circ} \mathrm{C}$. Supplementary Figure S1. Flow chart oflaboratory tests and six different direct antiglobulin test (DAT) methods performed with blood samples from dogs suspected to have immune-mediated hemolytic anemia. Supplementary Figure $\mathbf{S 2}$. Severity of anemia, reticulocytosis, hyperbilirubinemia, and free plasma hemoglobin concentration compared to microtiter plate direct antiglobulin test (MICRO DAT) results in dogs suspected to have immune-mediated hemolytic anemia. Supplementary Figure $\mathbf{S 3}$. Severity of anemia, reticulocytosis, bilirubinemia, and free plasma hemoglobin concentration results grouped based on $\geq 4$ DAT+ results in dogs suspected to have immune-mediated hemolytic anemia

\section{Acknowledgements}

Laboklin GmbH\&Co.KG kindly provided the working space, material, and assistance with their technical expertise. We thank the veterinary clinicians who submitted samples and the Laboklin team in Bad Kissingen, Germany for their assistance. The valuable statistical assistance by Dr. Hartnack as well as scientific editing by Dr. King are kindly acknowledged.

The authors would like to dedicate this article in honor of Jean Dodds, DVM, who undertook pioneering work in veterinary immunohematology, hemostasis, and transfusion medicine. She continues, at age 80 , to make major contributions to animal welfare and still heads the first non-for-profit canine blood bank Hemopet ${ }^{\circledR}$.

\section{Authors' contributions}

$\mathrm{NI}$ was performing all laboratory tests, gathered all data, statistically analyzed the data, and co-drafted the manuscript. JOZ and CNW were involved in setting up the immunodiagnostic tests in laboratory and contributed to the manuscript. EM was providing support for the entire study and contributed to the manuscript. UG developed the concept and study design, was setting up assays, performing data analyses, interpreting the data, and drafting the manuscript. All authors read and approved the final manuscript.

\section{Funding}

Alvedia, Limonest, France, kindly supplied the two in-clinic DAT kits. Laboklin GmbH\&Co.KG, Bad Kissingen, Germany, provided the workspace and other materials and equipment. The study design, collection, analyses, and interpretation of the data as well as the writing of manuscript were done independently.

\section{Availability of data and materials}

The datasets used and/or analyzed during the current study are available from the corresponding author on reasonable request.

\section{Declarations}

\section{Ethics approval and consent to participate}

The usage of left-over blood samples was approved by the Government in Bayern, Germany, and stated in the sample submission documentation of Laboklin GmbH\&Co.KG, Bad Kissingen, Germany.

\section{Consent for publication}

Not applicable.

\section{Competing interests}

This study is part of Nadine Idalan's doctoral thesis at the University of Zürich, Switzerland. Alvedia, Limonest, France, kindly offered their diagnostic kits for this study, but were not further involved in the project. The experiments were performed at Laboklin GmbH\&Co, Bad Kissingen, Germany, but independently and separately from the routine diagnostic service. Urs Giger has been a scientific advisor to Alvedia and Laboklin.

\section{Author details}

${ }^{1}$ Vetsuisse Faculty, University of Zürich, Winterthurerstrasse 260, 8057 Zürich, Switzerland. ${ }^{2}$ Laboklin GmbH\&Co.KG, Steubenstrasse 4, 97688 Bad Kissingen, Germany. ${ }^{3}$ Section of Medical Genetics, University of Pennsylvania, 3900 Delancey St, Philadelphia, PA 19104, USA.

Received: 12 April 2021 Accepted: 19 August 2021

Published online: 13 October 2021

\section{References}

1. Giger U. Regenerative anemias caused by blood loss or hemolysis. In: Ettinger SJ, Feldman EC, editors. Elsevier textbook of veterinary internal medicine. 2nd ed. St. Louis: Elsevier; 2006. p. 1886-908.

2. Giger U. Diagnosis of immune-mediated hemolytic anemia. Proceeding of ACVIM Forum, Maryland, USA. 2017. https://www.vin.com. Accessed 10 Apr 2021.

3. Caviezel LL, Raj K, Giger U. Comparison of 4 direct Coombs'test methods with polyclonal antiglobulins in anemic and nonanemic dogs for in-clinic or laboratory use. J Vet Intern Med. 2014. https://doi.org/10.1111/jvim. 12292.

4. Giger U, Idalan N, Caviezel L, Raj K. Controversies and updates on immunodiagnostics of IMHA in dogs. Proceeding of ACVIM Forum, 2021. https://www.vin.com. Accessed 1 July 2021.

5. Giger U. How I treat (diagnose): Immune-mediated hemolytic anemia. Proceedings of WSAVA Congress, Auckland, New Zealand. 2013. https:// www.vin.com. Accessed 10 Apr 2021.

6. Garden OA, Kidd L, Mexas AM, Chang YM, Jeffery U, Blois SL, Fogle JE, MacNeill AL, Lubas G, Birkenheuer A, Buoncompagni S, Dandrieux JRS, Di Loria A, Fellman CL, Glanemann B, Goggs R, Granick JL, LeVine DN, Sharp CR, Smith-Carr S, Swann JW, Szladovits B. ACVIM consensus statement on 
the diagnosis of immune-mediated hemolytic anemia in dogs and cats. J Vet Intern Med. 2019. https://doi.org/10.1111/jvim.15441.

7. MacNeill AL, Dandrieux J, Lubas G, Seelig D, Szladovits B. The utility of diagnostic tests for immune-mediated hemolytic anemia. Vet Clin Pathol. 2019. https://doi.org/10.1111/vcp.12771

8. Jäger U, Barcellini W, Broome CM, Gertz MA, Hill A, Hill QA, Jilma B, Kuter DJ, Michel M, Montillo M, Röth A, Zeerleder SS, Berentsen S. Diagnosis and treatment of autoimmune hemolytic anemia in adults: Recommendations from the First International Consensus Meeting. Blood Rev. 2020. https://doi.org/10.1016/j.blre.2019.100648.

9. Hill QA, Stamps R, Massey E, Grainger JD, Provan D, Hill A, Haematology, BSf. The diagnosis and management of primary autoimmune haemolytic anaemia. Br J Haematol. 2017. https://doi.org/10.1111/bjh.14478.

10. Moreschi C. Neue Tatsachen über die Blutkörperchenagglutinationen [New facts about blood cell agglutinations]. Zbl Bakt. 1908;456:46-9.

11. Sriwichai $C$, Jiraudommongkol M, Wutti-In Y, Vongchan P. Production of an anticanine globulin (polyspecific) reagent for laboratory investigation. J Small Anim Pract. 2011. https://doi.org/10.1111/j.1748-5827.2011. 01103.x.

12. Kaplan AV, Quimby FW. A radiolabeled staphylococcal protein A assay for detection of anti-erythrocyte lgG in warm agglutinin autoimmune hemolytic anemia of dogs and man. Vet Immunol Immunopathol. 1983. https://doi.org/10.1016/0165-2427(83)90042-9.

13. Widmann F, Barnes A, Case J, Garratty G, Holland P, McCullough JJ, Nusbacher J, Peterson EE, Steane SM, Wallace ME, Wells J. Technical manual. 8th ed. Washington DC: AABB; 1981. p. 422-516.

14. Barcellini W, Revelli N, Imperiali FG, Villa MA, Manera MC, Paccapelo C, Zaninoni A, Zanella A. Comparison of traditional methods and mitogen-stimulated direct antiglobulin test for detection of anti-red blood cell autoimmunity. Int J Hematol. 2010. https://doi.org/10.1007/ s12185-010-0578-9.

15. Rumsey DH, Ciesielski DJ. New protocols in serologic testing: a review of techniques to meet today's challenges. Immunohematology. 2000;16:131-7.

16. Fung MK, Grossman BJ, Hillyer CD, Westhoff CM. Technical manual. 18th ed. Maryland: AABB; 2014. p. 430-2.

17. Tan E, Bienzle D, Shewen P, Kruth S, Wood D. Potentially antigenic RBC membrane proteins in dogs with primary immune-mediated hemolytic anemia. Vet Clin Pathol. 2012. https://doi.org/10.1111/j.1939-165X.2011. 00391.x.

18. Wilkerson MJ, Davis E, Shuman W, Harkin K, Cox J, Rush B. Isotype-specific antibodies in horses and dogs with immune-mediated hemolytic anemia. JVet Intern Med. 2000. https://doi.org/10.1892/0891-6640(2000) 0142.3.co;2.

19. Woolhead VL, Szladovits B, Chan A, Swann JW, Glanemann B. Breed predispositions, clinical findings, and prognostic factors for death in dogs with nonregenerative immune-mediated anemia. J Vet Intern Med. 2021. https://doi.org/10.1111/jvim.15986.

20. Sun PL, Jeffery U. Effect of dilution of canine blood samples on the specificity of saline agglutination tests for immune-mediated hemolysis. JVet Intern Med. 2020. https://doi.org/10.1111/jvim.15945.

21. Wardrop K. Coombs'testing and its diagnostic significance in dogs and cats. Vet Clin North Am Small Anim Pract. 2012. https://doi.org/10.1016/j. cvsm.2011.09.005

22. Ebelt AK, Fuchs $S$, Weber $C$, Müller E, Giger U. Survey of blood groups DEA 1, DEA 4, DEA 5, Dal, and Kai 1/Kai 2 in different canine breeds from a diagnostic laboratory in Germany. Front Vet Sci. 2020. https://doi.org/10. 3389/fvets.2020.00085.

23. Lippi G, Caputo M, Banfi G, Daves M, Dolci A, Montagnana M, Miconi V, Milanesi B, Morandini M, Piva E, Salvagno GL, Troiano T, Giavarina D. Raccomandazioni di consenso SIBioC-SIMeL per la rilevazione e gestione dei campioni emolisati e utilizzo dell'indice di emolisi [Consensus SIBioC-SIMeL recommendations for the identification and management of haemolysed specimens and implementation of the haemolysis index]. Biochim Clin. 2011. https://doi.org/10.1007/ s13631-011-0022-z.

24. Mason N, Duval D, Shofer FS, Giger U. Cyclophosphamide exerts no beneficial effect over prednisone alone in the initial treatment of acute immune-mediated hemolytic anemia in dogs: a randomized controlled clinical trial. J Vet Intern Med. 2003. https://doi.org/10.1111/j.1939-1676. 2003.tb02435.x
25. Paes G, Paepe D, Meyer E, Kristensen AT, Duchateau L, Campos M, Daminet $\mathrm{S}$. The use of the rapid osmotic fragility test as an additional test to diagnose canine immune-mediated haemolytic anaemia. Acta Vet Scand. 2013. https://doi.org/10.1186/1751-0147-55-74.

26. Goggs R, Boag AK, Chan DL. Concurrent immune-mediated haemolytic anaemia and severe thrombocytopenia in 21 dogs. Vet Rec. 2008. https:// doi.org/10.1136/vr.163.11.323.

27. Chown B, Lewis M. The slanted capillary method of rhesus blood-grouping. J Clin Pathol. 1951. https://doi.org/10.1136/jcp.4.4.464.

28. Coombs RRA, Mourant AE, Race RR. A new test for the detection of weak and "incomplete" Rh agglutinins. Br J Exp Patho. 1945;26:255-66.

29. Slappendel R. The diagnostic significance of the direct antiglobulin test (DAT) in anemic dogs. Vet Immunol Immunopathol. 1979. https://doi. org/10.1016/0165-2427(79)90007-2.

30 Corp IBM, Released, . IBM SPSS statistics for windows, V.A. Armonk: IBM Corp; 2017.

31. Fleiss JR. Measuring nominal scale agreement among many raters. Psychol Bull. 1971. https://doi.org/10.1037/h0031619.

32. Landis JR, Koch GG. An application of hierarchical kappa-type statistics in the assessment of majority agreement among multiple observers. Biometrics. 1977. https://doi.org/10.2307/2529786.

33. Brennan P, Silman A. Statistical methods for assessing observer variability in clinical measures. BMJ. 1992. https://doi.org/10.1136/bmj.304.6840. 1491.

34. Shapiro SS, Wilk MB. An analysis of variance test for normality (for complete samples). Biometrics. 1965. https://doi.org/10.1093/biomet/52.3-4. 591.

35. Wilcoxin F. Probability tables for individual comparisons by ranking methods. Biometrics. 1947. https://doi.org/10.2307/3001946.

36. Student WSG. The probable error of a mean. Biometrics. 1908. https:// doi.org/10.1093/biomet/6.1.1.

37. Cochran WG. The Chi-square test of goodness of fit. Ann Math Stat. 1952. https://doi.org/10.1214/aoms/1177729380.

38. Fisher RA. On the interpretation of $\times 2$ from contingency tables, and the calculation of P. J R Stat Soc. 1922. https://doi.org/10.2307/2340521.

39. Saah AJ, Hoover DR. "Sensitivity" and "specificity" reconsidered: the meaning of these terms in analytical and diagnostic settings. Ann Intern Med. 1997. https://doi.org/10.7326/0003-4819-126-1-19970 1010-00026.

40. Parikh R, Mathai A, Parikh S, Chandra Sekhar G, Thomas R. Understanding and using sensitivity, specificity and predictive values. Indian J Ophthalmol. 2008. https://doi.org/10.4103/0301-4738.37595.

41. Giger U. Diagnosis of Hemolytic Anemias. Proceedings of WSAVA Congress, Vancouver, Canada. 2001. https://www.vin.com. Accessed 10 Apr 2021.

42 Warman SM, Murray JK, Ridyard A, Eastwood J, Silva S, Day MJ. Pattern of Coombs' test reactivity has diagnostic significance in dogs with immune-mediated haemolytic anaemia. J Small Anim Pract. 2008. https://doi.org/10.1111/j.1748-5827.2008.00641.X.

43. Weinkle TK, Center SA, Randolph JF, Warner KL, Barr SC, Erb HN. Evaluation of prognostic factors, survival rates, and treatment protocols for immune-mediated hemolytic anemia in dogs: 151 cases (1993-2002). J Am Vet Med Assoc. 2005. https://doi.org/10.2460/javma.2005.226. 1869.

44. Burgess K, Moore A, Rand W, Cotter SM. Treatment of immune-mediated hemolytic anemia in dogs with cyclophosphamide. J Vet Intern Med. 2000. https://doi.org/10.1892/0891-6640(2000)014\%3c0456: toihai\%3e2.3.co;2.

45. Miller SA, Hohenhaus AE, Hale AS. Case-control study of blood type, breed, sex, and bacteremia in dogs with immune-mediated hemolytic anemia. J Am Ved Med Assoc. 2004. https://doi.org/10.2460/javma. 2004.224.232.

46. Klag AR, Giger U, Shofer FS. Idiopathic immune-mediated hemolytic anemia in dogs: 42 cases (1986-1990). J Am Vet Med Assoc. 1993;202:785-8.

47. Werner LL. Coombs' positive anemias in the dog and cat. Comp Cont Educ Pract Vet. 1980;2:96-101.

48. Carr AP, Panciera DL, Kidd L. Prognostic factors for mortality and thromboembolism in canine immune-mediated hemolytic anemia: a retrospective study of 72 dogs. J Vet Intern Med. 2002. https://doi.org/10.1892/ 0891-6640(2002)016\%3c0504:pffmat\%3e2.3.co;2. 
49. Whipple KM, Leissinger MK, Beatty SS. Frequency and classification of errors in laboratory medicine at a veterinary teaching hospital in the United States. Vet Clin Pathol. 2020. https://doi.org/10.1111/vcp.12851.

50. Lippi G, Salvagno GL, Montagnana M, Brocco G, Guidi GC. Influence of hemolysis on routine clinical chemistry testing. Clin Chem Lab Med. 2006. https://doi.org/10.1515/CCLM.2006.054.

51. Désidéri-Vaillant C, Oye G, Appéré G, Carassou-Beauvais P, Pérennec V, Caron S, Sapin J, Corbé H, Berna A. Evaluation of the effect of hemolysis, bilirubin, lipemy, glucosis and natrium on mean concentration of hemoglobin. Ann Biol Clin. 2018. https://doi.org/10.1684/abc.2018.1376.

52 Zandecki M, Genevieve F, Gerard J, Godon A. Spurious counts and spurious results on haematology analysers: a review. Part II: white blood cells, red blood cells, haemoglobin, red cell indices and reticulocytes. Int J Lab Hematol. 2007. https://doi.org/10.1111/j.1365-2257.2006.00871.x.

53. Assenmacher TD, Jutkowitz LA, Koenigshof AM, Lucidi CA, Scott MA. Clinical features of precursor-targeted immune-mediated anemia in dogs: 66 cases (2004-2013). J Am Vet Med Assoc. 2019. https://doi.org/10.2460/ javma.255.3.366.

54. Lucidi CA, de Rezende CLE, Jutkowitz LA, Scott MA. Histologic and cytologic bone marrow findings in dogs with suspected precursor-targeted immune-mediated anemia and associated phagocytosis of erythroid precursors. Vet Clin Pathol. 2017. https://doi.org/10.1111/vcp.12502.

55. Weiss DJ. Bone marrow pathology in dogs and cats with non-regenerative immune-mediated haemolytic anaemia and pure red cell aplasia. J Comp Pathol. 2008. https://doi.org/10.1016/j.jcpa.2007.10.001.

56. Rothuizen J, van den Brom WE, Fevery J. The origins and kinetics of bilirubin in dogs with hepatobiliary and haemolytic diseases. J Hepatol. 1992. https://doi.org/10.1016/0168-8278(92)90006-b.

57 Peterson J. Hematology/Oncology. In: Birchard SJ, Sherding RG, editors. Sauders manual of small animal practice. 3rd ed. Elsevier: Amsterdam; 2006. p. 238. https://doi.org/10.1016/B0-7216-0422-6/X5001-3.

58. Slappendel RJ, van Zwieten R, van Leeuwen M, Schneijdenberg CT. Hereditary spectrin deficiency in Golden Retriever dogs. J Vet Intern Med. 2005. https://doi.org/10.1892/0891-6640(2005)19\%3c187:hsdigr\%3e2.0. $\mathrm{co} 2$.

59. Petkova-Kirova P, Hertz L, Danielczok J, Huisjes R, Makhro A, Bogdanova A, Mañú-Pereira MDM, Vives Corrons JL, van Wijk R, Kaestner L. Red blood cell membrane conductance in hereditary haemolytic anaemias. Front Physiol. 2019. https://doi.org/10.3389/fphys.2019.00386.

60. Weiss D, Tvedten H. Erythrocytes disorders. In: Small animal clinical diagnosis by laboratory methods, 5th ed, Willard; Tvedten H. Elsevier. 2012. p 50. doi:https://doi.org/10.1016/C2009-0-59996-5.

61. Overmann JA, Sharkey LC, Weiss DJ, Borjesson DL. Performance of 2 microtiter canine Coombs' tests. Vet Clin Path. 2007. https://doi.org/10. 1111/j.1939-165x.2007.tb00205.x.

62. Giger U. Letter regarding "Effect of dilution of canine blood samples on the specificity of saline agglutination tests for immune-mediated hemolysis," original and modified saline agglutination tests vs direct Coombs' tests. J Vet Intern Med. 2021. https://doi.org/10.1111/jvim.16130.

63. Segel GB, Lichtman MA. Direct antiglobulin ("Coombs") test-negative autoimmune hemolytic anemia: a review. Blood Cells Mol Dis. 2014. https://doi.org/10.1016/j.bcmd.2013.12.003.
64. Wardrop KJ. The Coombs' test in veterinary medicine: past, present, future. Vet Clin Path. 2005. https://doi.org/10.1111/j.1939-165x.2005 tb00057.x.

65. Morley P, Mathes M, Guth A, Dow S. Anti-erythrocyte antibodies and disease associations in anemic and nonanemic dogs. J Vet Intern Med. 2008. https://doi.org/10.1111/j.1939-1676.2008.0112.x.

66. Piek CJ. Canine idiopathic immune-mediated haemolytic anaemia: a review with recommendations for future research. Vet Q. 2011. https:// doi.org/10.1080/01652176.2011.604979.

67. Piek CJ, Teske E, van Leeuwen MW, Day MJ. Good agreement of conventional and gel-based direct agglutination test in immune-mediated haemolytic anaemia. Acta Vet Scand. 2012. https://doi.org/10.1186/ 1751-0147-54-10.

68. Quigley KA, Chelack BJ, Haines DM, Jackson ML. Application of a direct flow cytometric erythrocyte immunofluorescence assay in dogs with immune-mediated hemolytic anemia and comparison to the direct antiglobulin test. J Vet Diagn Invest. 2001. https://doi.org/10.1177/10406 3870101300403.

69. Seth M, Jackson KV, Winzelberg S, Giger U. Comparison of gel column, card, and cartridge techniques for dog erythrocyte antigen 1.1 blood typing. Am J Vet Res. 2012. https://doi.org/10.2460/ajvr.73.2. 213.

70. Titlestad K, Georgsen J, Andersen H, Kristensen T. Detection of irregular red cell antibodies: more than 3 years of experience with a gel technique and pooled screening cells. Vox Sang. 1997. https://doi.org/10.1046/j. 1423-0410.1997.7340246.x.

71. Springer NL. Flow cytometric detection of Anti-RBC antibodies. Proceeding of ACVIM Forum. 2021. https://www.vin.com. (Accesseed 1 July 2021)

72. Wilkerson MJ. Principles and applications of flow cytometry and cell sorting in companion animal medicine. Vet Clin North Am Small Anim Pract. 2012. https://doi.org/10.1016/j.cvsm.2011.09.012.

73. Greene CE, Kristensen F, HoffEJ, Wiggins MD. Cold hemagglutinin disease in a dog. J Am Vet Med Assoc. 1977;170:505-10.

74. Day MJ. Serial monitoring of clinical, haematological and immunological parameters in canine autoimmune haemolytic anaemia. J Small Anim Pract. 1996. https://doi.org/10.1111/j.1748-5827.1996. tb02313.x.

75. Hohenhaus AE. Importance of blood groups and blood group antibodies in companion animals. Transfus Med Rev. 2004. https://doi.org/10.1016/j. tmrv.2003.12.003.

76. Herter L, Weingart C, Bock N, Merten N, Kohn B. Alloimmunisierung bei Hunden infolge einer transfusion. Tierarztl Prax Ausg K Kleintiere Heimtiere. 2021. https://doi.org/10.1055/s-0040-1722398.

77. Piek CJ, Junius G, Dekker A, Schrauwen E, Slappendel RJ, Teske E. Idiopathic immune-mediated hemolytic anemia: treatment outcome and prognostic factors in 149 dogs. J Vet Intern Med. 2008. https://doi.org/10. 1111/j.1939-1676.2008.0060.x.

\section{Publisher's Note}

Springer Nature remains neutral with regard to jurisdictional claims in published maps and institutional affiliations.

\footnotetext{
Ready to submit your research? Choose BMC and benefit from:

- fast, convenient online submission

- thorough peer review by experienced researchers in your field

- rapid publication on acceptance

- support for research data, including large and complex data types

- gold Open Access which fosters wider collaboration and increased citations

- maximum visibility for your research: over 100M website views per year
}

At BMC, research is always in progress.

Learn more biomedcentral.com/submissions 